\title{
Ethanol catalytic membrane reformer for direct PEM FC feeding
}

Reinhold Koch a,b,c, ${ }^{\star}$, Eduardo López ${ }^{\text {a,d }}$, Núria J. Divins ${ }^{a}$, Miguel Allué ${ }^{b}$, Andreas Jossen ${ }^{c}$, Jordi Riera ${ }^{b}$, Jordi Llorca ${ }^{a}$

${ }^{a}$ Institut de Tècniques Energètiques, Universitat Politècnica de Catalunya, Diagonal 647, Ed. ETSEIB, 08028 Barcelona, Spain

${ }^{\mathrm{b}}$ Institut de Robòtica i Informàtica Industrial (UPC-CSIC), Llorensi Artigas 4-6, 08028 Barcelona, Spain

${ }^{c}$ Institute for Electrical Energy Storage Technology, Technische Universität München, Karlstr. 45, 80333

Munich, Germany

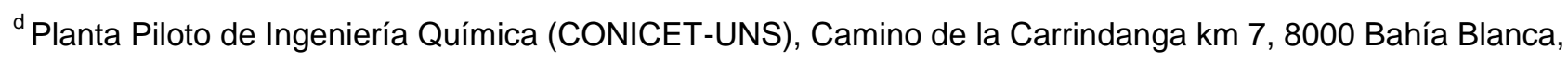
Argentina

\section{* Corresponding author:}

Reinhold Koch

Technische Universität München

Institute for Electrical Energy Storage Technology

Karlstr. 45

80333 Munich

Germany

Tel.: 00498928926972

Fax.: 00498928926968

e-mail: reinhold.koch@tum.de 


\section{ABSTRACT}

In this paper an ethanol reformer based on catalytic steam reforming with a catalytic honeycomb loaded with $\mathrm{RhPd} / \mathrm{CeO}_{2}$ and palladium separation membranes with an area of $30.4 \mathrm{~cm}^{2}$ has been used to generate a pure hydrogen stream of up to $100 \mathrm{ml} / \mathrm{min}$ to feed a PEM fuel cell with an active area of $5 \mathrm{~cm}^{2}$. The fuel reformer behavior has been extensively studied under different temperature, ethanol-water flow rate and gas pressure at a fixed S/C ratio of 1.6 (molar). The hydrogen yield has been controlled by acting upon the ethanol-water fuel flow and gas pressure.

A mathematical model of the ethanol reformer has been developed and an adaptive and predictive control has been implemented on a real time system to take account of its nonlinear behavior. With this control the response time of the reformer can be reduced by a factor of 7 down to 8 seconds.

The improved dynamics of the controlled reformer match better the quickly changing hydrogen demands of fuel cells. They reached a magnitude where costly hydrogen buffers between the reformer and the fuel cell can be omitted and an electric buffer at the output of the fuel cell is sufficient.

\section{KEYWORDS}

Ethanol steam reforming, Metal membrane, PEM Fuel Cell, Sensitivity analysis, Dynamic modeling, Reformer Control 


\section{INTRODUCTION}

The conventional propulsion by an internal combustion engine in the automotive industry is no longer considered as being the only option. Poor efficiency, high emission rates and dependence on oil make it unattractive. An electric vehicle driven by an electric motor and fed by a battery is praised as the technology of the future. But this solution also implies several economic and technical problems mainly because the battery cells are still too expensive, too heavy and charging takes too much time. In city traffic, it will certainly prevail, because the elimination of emissions is particularly good for the air quality in the cities. But for long distance rides, for trucks and coaches, the battery will either limit the range too much or will take up too much space in the vehicle. If the battery development is extrapolated from the past into the future, it is not to assume that the specific capacity of a battery will significantly increase in the next few decades.

The combustion of hydrogen is one of the cleanest ways to obtain a high amount of energy. Furthermore, its conversion inside a fuel cell to electrical energy yields the highest efficiency known today. The development of fuel cells has advanced in the last years and could be used for several applications: a fuel cell vehicle is just one of them. The greatest obstacle for the wide use of hydrogen as an energy carrier in our daily life is the generation and distribution of hydrogen. Although hydrogen can be produced in various ways, most of the hydrogen produced today comes from large chemical plants making its way to the final customer very long. Due to the lack of a proper way for hydrogen storage, its production should take place right at the refilling station or even in the vehicle itself making hydrogen storage unnecessary. Reforming technologies are suited for this purpose [1]. In a reforming process, a gaseous or liquid fuel is mixed with steam (steam reforming) or with a steam/air mixture (oxidative reforming) over an appropriate catalyst to yield mostly hydrogen and carbon dioxide, although minor amounts of carbon monoxide, methane and other light hydrocarbons are always present. However, hydrogen for low-temperature fuel cells can only be used if it fulfills high purity demands; even small amounts of carbon monoxide poison the hydrogen reaction at the anode of the fuel cell, resulting in a lower fuel cell potential and, consequently in a lower energy conversion efficiency. Therefore, the gas streams of fuel reformers have to be purified before being used in this type of fuel cells. Among the different methods to accomplish this (pressure washing, pressure swing adsorption, cryogenic distillation, membrane separation and catalytic purification), only membrane separation and catalytic purification are suited for on-board scenarios [1].

Recently, we have built a catalytic ethanol membrane reformer towards the simultaneous production and separation of pure hydrogen. The unit was successfully tested; achieving reaction yields of $3.1 \mathrm{~mol}$ hydrogen generated per mol of ethanol in the feed with hydrogen recuperation values up to $70 \%$ [2]. Steam reforming of ethanol was chosen due to its high efficiency and the possibility to apply the process at a small scale. In addition, bio-ethanol production ramped up in the last years, is safe, easy to handle, and its use may be considered $\mathrm{CO}_{2}$-neutral. In this work, we have modeled the catalytic ethanol membrane reformer and implemented a control system. We have also tested on-line the reformer with a PEM fuel cell. 


\section{CATALYTIC ETHANOL MEMBRANE REFORMER}

\subsection{Description}

The ethanol reformer consists of two stages as shown in Fig. 1. The first step produces a reformed gas with a high hydrogen content, starting from an ethanol-water mixture over catalytic honeycombs loaded with $\mathrm{RhPd} / \mathrm{CeO}_{2}$ in a tubular reactor [2]. The three main reactions, which occur consecutively during the reforming process, are decomposition of ethanol (eq. 1), the water gas shift reaction (eq. 2) and the reforming of methane (eq. 3) [3].

$$
\begin{array}{ll}
\mathrm{CH}_{3} \mathrm{CH}_{2} \mathrm{OH} \rightarrow \mathrm{CH}_{4}+\mathrm{CO}+\mathrm{H}_{2} & \Delta \mathrm{H}=+50 \mathrm{~kJ} \mathrm{~mol}-1^{-1} \\
\mathrm{CO}+\mathrm{H}_{2} \mathrm{O} \leftrightarrows \mathrm{CO}_{2}+\mathrm{H}_{2} & \Delta \mathrm{H}=-41 \mathrm{~kJ} \mathrm{~mol}^{-1} \\
\mathrm{CH}_{4}+\mathrm{H}_{2} \mathrm{O} \leftrightarrows \mathrm{CO}+3 \mathrm{H}_{2} & \Delta \mathrm{H}=+206 \mathrm{~kJ} \mathrm{~mol}^{-1}
\end{array}
$$

In the second step, a palladium-based selective membrane (Reb Research \& Consulting) separates the hydrogen from the rest of the reformed gas. The hydrogen is over $99.999 \%$ pure (checked by gas chromatography), satisfying even the rigorous purity demands of a PEM fuel cell.

Fig. 1. Scheme of the reformer processes.

The details of the reformer and initial measurements were described in [2]. In this work we have implemented automated valves and sensors in order to set the reformer in a proper control environment. The current design of the reformer (Fig. 2) consists of a fuel tank and a fuel pump (Knauer Smartline HPLC). The liquid is pumped into the reformer core, which is maintained at a constant temperature by means of an electrical heating resistance. The temperature is measured with a thermocouple and is controlled by a PID controller (Fuji PXR4) as well as a solid state relay. The ethanol-water fuel first evaporates and enters the catalytic zone where it reforms to a mixture of hydrogen, methane, carbon monoxide and carbon dioxide. The reformed gas immediately enters the second stage in the same tubular reactor, where four Pd-Ag deadend membrane tubes (total membrane area of $30.4 \mathrm{~cm}^{2}$ ) separate part of the hydrogen from the rest of the reformed gas. After separation, the waste gas and the pure hydrogen streams leave the reformer core in different conduits through check valves and pass through water traps. The waste gas also passes through a particle filter, while the pure hydrogen gas is already particle-free because it has been filtered by the metallic membrane. Additional thermocouples were placed at different locations to control temperature gradients and isolation.

The membrane separation process is driven by a pressure gradient. The pressure of the waste gas outlet is controlled by a pressure controller, which consists of a pressure meter with a separated valve (Bronkhorst 
EL-Press P-702CV). The pressures at the waste gas outlet and inside the reformer core are assumed to have the same value. From now on, the term "waste gas pressure $\left(P_{W G}\right)$ " will refer to this pressure. The pressure on the pure hydrogen side is left at ambient pressure. The hydrogen flow rate is measured by a flow meter (Bronkhorst F-111B). The pure hydrogen and the waste gas effluents are monitored on line with an Agilent 3000A micro-GC equipped with PLOT U, Stabilwax and $5 \AA$ Molsieve columns.

Fig. 2. Scheme of the ethanol catalytic membrane reformer.

\subsection{Operation Characteristics}

The ethanol reformer can be controlled by four main variables: temperature, fuel mixture composition (steam-to-carbon ratio, $\mathrm{S} / \mathrm{C}$ ), pressure and fuel flow rate. The theoretical impact of the first three of the four parameters can be easily explained by the Le Châtelier's principle taking into account the overall reaction (eq. 4).

$$
\mathrm{CH}_{3} \mathrm{CH}_{2} \mathrm{OH}+3 \mathrm{H}_{2} \mathrm{O} \rightarrow 2 \mathrm{CO}_{2}+6 \mathrm{H}_{2} \quad \Delta \mathrm{H}=+174 \mathrm{~kJ} \mathrm{~mol}^{1}
$$

Since the reforming process is endothermic, an increase in temperature shifts the reaction to the product side as do $\mathrm{S} / \mathrm{C}$ ratios exceeding the stoichiometric value of $1.5\left(\mathrm{CH}_{3} \mathrm{CH}_{2} \mathrm{OH}: \mathrm{H}_{2} \mathrm{O}=1: 3\right)$. The separation performance of the metallic membrane is not significantly affected by temperature. $\mathrm{S} / \mathrm{C}$ values greater than 1.5 limit the carbon deposition on the catalyst surface, but at the same time increases the amount of energy needed to heat and evaporate the additional water. In our previous study [2] we encountered the best operational value to get the highest hydrogen flow rate at $S / C=1.6$, and this is the value used in this work, which happens to equal a volumetric ratio of $1: 1$ for the ethanol-water mixture.

The pressure effect is not straightforward since a change of pressure causes two opposite effects. On one hand, the reaction is progressively inhibited as the pressure of the reactor increases but, on the other hand, the hydrogen separation in the metallic membrane improves considerably following the Sievert's law (related to the hydrogen partial pressure at both sides of the membrane, see below). Finally, the fuel flow rate commands obviously the total hydrogen flow rate, since the more fuel is provided the more reformed gas is produced within certain conditions (no membrane and catalyst limitations). At a fixed temperature and $\mathrm{S} / \mathrm{C}$ ratio, pressure and fuel flow rate are the two variables to control during the ethanol reformer operation.

\section{REFORMER BEHAVIOR}

\subsection{Static Response}

\subsubsection{Catalytic reaction}


The ethanol reformer was tested at temperatures of $873 \mathrm{~K}$ and $923 \mathrm{~K}$ and at several different flow rates and pressures. In accordance to the preceding section, the hydrogen yield at $873 \mathrm{~K}$ was much lower than that at $923 \mathrm{~K}$. Furthermore, at $873 \mathrm{~K}$ the hydrogen flow was not stable under high fuel flow rates. Therefore, further work was carried out at a fixed temperature of $923 \mathrm{~K}$.

Fig. 3(a) shows the selectivity attained at this temperature and 10 bar in the catalytic stage under different fuel flow rates and total ethanol conversion. An increase of the flow rate results in a decrease of the contact time between reactants (water and ethanol) and the catalyst, which negatively affects the water gas shift equilibrium (eq. 2) and less carbon monoxide is transformed into $\mathrm{CO}_{2}$.

Fig. 3(b) shows the distribution of products obtained at the same temperature under different pressures at a fixed value of $250 \mu \mathrm{l} / \mathrm{min}$ of fuel flow rate (total ethanol conversion). In this case, the pressure has a strong influence on the hydrogen yield, the higher the pressure the lower the selectivity towards hydrogen. The effect of pressure on the reaction is related to the variation of moles between products and reactants. Since in the complete reaction (eq. 4) the moles of products double the moles of reactants, an increase of pressure inhibits the reaction; and the reforming of methane (eq. 3), which is the last step of the reforming process, is progressively shifted to the reactants side.

Fig. 3. Selectivity on a dry basis obtained at: (a) $923 \mathrm{~K}$ and $P_{W G}=10$ bar under different fuel flow rates; (b) at $923 \mathrm{~K}$ and $F_{\text {Fuel }}=250 \mu \mathrm{l} / \mathrm{min}$ under different pressures.

\subsubsection{Membrane separation}

The hydrogen selective separation stage is a set of four $\mathrm{Pd}-\mathrm{Ag}$ metallic membranes, which permeate only the hydrogen leaving the rest of the gas on the reformer side. The mass transfer mechanism can be expressed using the Sievert's law (eq. 5).

$$
Q=\frac{P_{e}}{\delta} \cdot A \cdot\left(\sqrt{P_{W G}}-\sqrt{P_{H 2 \_p u r e}}\right)
$$

In Fig. 4. the separation capability of the membranes $\left(\% \mathrm{H}_{2}\right.$ permeated with respect to $\mathrm{H}_{2}$ produced in the catalytic stage) at different waste gas pressure and fuel flow rate values is shown. The hydrogen output pressure was left at ambient pressure. It can be seen that at high pressures the separation capability of the membrane increases almost linearly with the waste gas pressure. At low waste gas pressures this behavior changes and the separation capability decreases significantly. The measured values confirm the Sievert's law, since the square root function becomes quite linear at higher argument values and is very nonlinear at lower argument values. At higher flow rates the separation efficiency decreases since the gas flow passes too quickly for the membrane to separate the hydrogen properly due to the physical limitation of the membrane surface area. 
Fig. 4. Hydrogen separation efficiency of the membrane stage measured at $923 \mathrm{~K}$ under different fuel flow rates and pressures.

\subsection{Dynamic Response}

\subsubsection{Catalytic reaction}

The response to a change of fuel flow rate had a small dead time of 1 to 5 seconds. Unfortunately, no dependency on the operating conditions could be identified. Taking into account the experimental data, a dead time of 2 seconds was assumed for control purposes. The response after the dead time ending with the new fuel flow rate followed a First Order System type behavior (eq. 6).

$$
F_{H 2}=\Delta F_{H 2} \cdot e^{-t / \tau_{F F}}
$$

The time constant $\tau_{F F}$ can be considered as the main dynamic time constant of the ethanol reformer and it is in the scope of 10 to 50 seconds as long as the flow rate of the hydrogen output is not lower than 10 $\mathrm{ml} / \mathrm{min}$ (Fig. 5). Operating below this limit would cause the reformer to become almost uncontrollable because the time constant becomes very large. For modeling purposes, the reverse value was used, which was approximated by a linear function with the parameters hydrogen flow rate and waste gas pressure.

Fig. 5. Variation of the dynamic time constant of the reformer at different hydrogen flow rates and waste gas pressures as used in the reformer model.

\subsubsection{Membrane separation}

The reformer showed an interesting dynamic behavior when the waste gas pressure was varied. When the waste gas pressure was decreased, a hydrogen peak could be noticed at the hydrogen outlet. On the contrary, if the waste gas pressure was increased, the hydrogen output showed a negative peak, which was much smaller than its positive equivalent resulting into a non-symmetrical response. To understand this behavior, the hydrogen partial pressure gradient inside the membrane separation stage of the reformer has to be considered.

At static conditions, the partial pressure of hydrogen decreases along the membrane with an exponential function, as shown in Fig. 6(a). Due to a higher hydrogen concentration, the upper part of the membrane contributes much more to the pure hydrogen flow than the lower part. If the waste gas pressure is suddenly decreased, the valve of the pressure controller releases a high amount of gas. It can be assumed that the hydrogen poor gas at the lower part of the membrane is pushed outwards and the hydrogen rich gas, which was still above the membrane, is now pushed downwards. With this sudden change, the hydrogen partial pressure gradient changes to an almost constant distribution along the membrane, as shown in Fig. $6(\mathrm{~b})$. 
Now, the lower part of the membrane contributes much more to the separation process and a hydrogen peak is obtained until a new static hydrogen partial pressure gradient is established.

In contrast, if the waste gas pressure is increased, the waste gas valve closes allowing less gas to exit the system and thus staying inside the reformer. However, this waste gas is hydrogen-poor and dilutes the gas inside the reformer, which results in a steeper hydrogen partial pressure gradient and hence a negative hydrogen peak. Far from being a limitation, we have conveniently used this transient behavior in the outlet hydrogen flow to decrease the response time of the reformer.

Fig. 6. Schematic explanation of the formation of a transient hydrogen peak following a decrease in the waste gas pressure of the reformer (see text for details).

Fig. 7. Example for hydrogen peak at pressure decrease

Fig. 7 shows the shape of a hydrogen peak following a pressure decrease from P1 to P2. The time $T_{P k}$ (Tpeak) at the maximum of the hydrogen peak can be approximated by a linear function with the waste gas pressure and fuel flow rate as variables (Fig. 8(a)). However, the absolute value of the hydrogen peak, $H 2_{P k}$ (H2peak), cannot be adequately approximated by a linear function and a quadratic function has been used (Fig. 8(b)). Again, for modeling purposes and as we did for the time constant $\tau_{F F}$ of the catalytic reaction, the inverted values have been used to normalize each hydrogen peak. The resulting normalized curves are shown in Fig. 9. They were approximated by a third order polynomial.

Fig. 8. Linear approximation of $T_{P k}(\mathrm{a})$ and quadratic approximation of $H 2_{P k}(\mathrm{~b})$.

Fig. 9. Pressure response curves with third order polynomial approximation.

When both characteristics, the catalyst and the membrane, are combined, the hydrogen yield over the whole operating range becomes quite linear and can be approximated by a simple linear function, as shown in Fig. 10. At low pressures, the reforming activity works better and compensates for the poor separation efficiency. At high pressures, the separation works better and compensates for the decrease in reformed hydrogen performance.

Fig. 10. Hydrogen yield by the combination of catalytic reforming with membrane separation 
These two effects balance each other in a wide range of pressure values and result in a broad optimum plateau at which a high hydrogen yield can be obtained. For medium fuel flow rates, between 100 and 300 $\mathrm{ml} / \mathrm{min}$, the maximum can be found at 10 bars.

\section{DYNAMIC MODEL}

A complete dynamic model of the reformer must take into account that the total hydrogen output depends on two mechanisms that can be modeled by interconnected sub-models.

The first sub-model takes into consideration that the static hydrogen output flow depends on the fuel flow rate and on the waste gas pressure at the given temperature of $923 \mathrm{~K}$ and an $\mathrm{S} / \mathrm{C}$ ratio of 1.6 (see section 2.2. ). The dynamic transition to changes in the input operational conditions takes place according to a first order system behavior with a variable time constant, as described in section 3.2.1. In this sub-model the waste gas flow is also modeled with the same dynamic behavior as the hydrogen flow. A block diagram of this sub-model can be found in Fig. 11.

Fig. 11. Reformer sub-model 1: response to a fuel flow change

The second sub-model calculates the additional hydrogen that is obtained when the waste gas pressure is decreased, as described in section 3.2.2. This sub-model is more complex, as shown in Fig. 12. It basically represents the behavior of the decentralized PI controller for the waste gas pressure and that of the hydrogen selective membrane. Initially, the pressure set point passes through a signal conditioning filter and is used to calculate the pressure error.

Fig. 12. Reformer sub-model 2: response to waste gas pressure change

This error is used by the PI controller to adjust the valve opening in order to establish an output flow rate that maintains the desired pressure. The delay of the valve is described by a separate first order system block. Finally, in this sub-model, the flow rate of the gas which leaves the reformer is subtracted from the produced waste gas. Subsequently the flows difference either increases or decreases the waste gas pressure inside the reformer although its maximum value is limited to the produced waste gas flow rate. An anti-windup has been implemented but excluded from the diagram to maintain its clarity. As in static conditions the additional hydrogen produced by a decrease in the waste gas pressure must be zero, only the difference between the produced gas and the gas leaving the reformer is considered. This difference is already peak shaped but in order to obtain a peak, similar to the one in Fig. 7, the peak time is adjusted with a second order model (with $T_{P T 1}$ and $T_{P T 2}$ ) and the peak value is calibrated using the quadratic function described in Fig. $8(\mathrm{~b})$ having the waste gas pressure $P_{W G}$ and the fuel flow rate $F_{\text {Fuel }}$ as input. 


\section{REFORMER CONTROLLER}

The controller performs two main control actions which correspond to the two control variables: fuel flow rate and waste gas pressure. While the fuel flow rate is the most important variable, actuating on the pressure during dynamic load changes improves the system response time.

Fig. 13. Controller action schematics: (a) fuel flow rate control action, (b) pressure control action

\subsection{Fuel Flow Rate Controller}

The fuel flow rate controller implements three control actions as shown in Fig. 13(a): feed-forward, proportional and integral. The feed-forward control action uses the Hydrogen yield relation of Fig. 10 to estimate the fuel flow rate which is necessary to obtain the desired hydrogen flow rate. It is complemented with the proportional and integral actions [4].

The integral action provides accuracy to the controlled variable. A convenient time constant $T_{N}\left(=1 / K_{l}\right)$ for this action depends on the time constant $\tau_{F F}$ of the reformer: rather small $T_{N}$-values can be used when the reformer dynamics are fast and larger ones are necessary to ensure stability if the reformer dynamics is slow.

For the reformer application, as $\tau_{F F}$ can be estimated directly from the experimental relation shown in Fig. 5 , it was found that a convenient value for the integral action constant $K_{l}$ is $\left(2 \times \tau_{F F}\right)^{-1}$. The effect of this Integral action alone with a moderate Proportional part $\left(K_{P}=5\right)$ would result in a system response of around 60 seconds.

If the PI-control is combined with the feed-forward action, the system response time decreases significantly to only about 12 seconds, but with the cost of a high overshoot (see Fig. 14, H2, FF+PI). This happens because the dead time of the system is not fully compensated and the integral part of the controller is quite strong. To reduce the time response two additional effects have been introduced: a) a Smith Predictor to account for the dead time and to avoid high fuel flow rate peaks and b) the proportional coefficient $\left(K_{P}^{\prime} \times \tau_{F F} \times K_{H_{2}{ }_{2} F F}\right.$ with $\left.K_{P}^{\prime}=2\right)$ is made adaptively stronger by multiplying it by the system time constant $\tau_{F F}$ with the system gain $K 3_{H_{2} F F}$, which applies when the fuel flow rate is changed, allowing the proportional part to be greater at lower system dynamics and smaller at higher system dynamics. In this way the proportional action of the controller compensates the system dynamics and almost assures a constant system response time throughout the entire operational range. With this control, the system response time decreases to about 10 seconds (see Fig. 14, H2, FF+l+FCP).

\subsection{Pressure Controller}

The pressure controller assists the control action on the fuel flow rate as a second control variable [5]; see Fig. 13(b). The pressure controller acts only if the error of the set point to the actual hydrogen flow rate is bigger than $1.5 \mathrm{ml} / \mathrm{min}$ and in this case predicts the amount of hydrogen which will additionally leave the 
reformer in the future at time $T_{P k}$. The increase in hydrogen output in the future can come from two different sources: an increase of fuel flow $\left(F_{\mathrm{H}_{2} F \mathrm{FF}}(t)\right)$, which depends on the chosen fuel flow controller configuration and tuning, and from a previous pressure decrease $\left(F_{H 2} P(t)\right)$. The estimated effect of a previous pressure decrease is calculated according to Fig. 8(b). and then stored on line during system operation in a history memory. All these stored estimations of additional hydrogen output is convoluted with the normalized pressure decrease response function of Fig. 9. By using this normalized response function only the peak value of the estimated hydrogen increase has to be stored at the time the pressure is decreased. Decreasing the pressure to obtain more hydrogen flow has a specific dead time $T_{P k}$ before the peak of the additional hydrogen occurs. The hydrogen prediction is evaluated at $T_{P k}$ (black circle in Fig. 13(b)) and the expected value is subtracted from the current hydrogen error resulting in the hydrogen error of the future at the time $T_{P k}$. The pressure decrease necessary for $1 \mathrm{ml} / \mathrm{min}$ of hydrogen flow can be calculated according to the operating conditions $P_{W G}$ and $F_{F u e l}$. This value is multiplied by the hydrogen error at time $T_{P k}$ and the necessary waste gas pressure decrease is obtained.

The pressure controller action relies on a proper system model and can only act as long as the pressure stays within a specific range. The pressure controller makes sure that, according to the hydrogen set point, the pressure is not decreased too far. When the fuel flow controller action and the pressure controller action are used, the response time is further reduced to 8 seconds (see Table $1, H 2, F F+l+F C P+P B$ ). An overall comparison of all control types behavior is shown in Fig. 14 and in Table 1.

Fig. 14. Dynamic response of the controlled reformer for a step from 34 to $57 \mathrm{ml} / \mathrm{min}$ of $\mathrm{H}_{2}$

Table 1. Performance of different controllers 


\section{ETHANOL REFORMER AND PEM FUEL CELL}

The hydrogen supplied by the reformer is of high purity and is produced with a fast time response to set point changes. Hence, the reformer is convenient to supply hydrogen to a PEM fuel cell with no side effects like fuel cell catalyst poisoning produced by impurities or fuel shortage. When connected there is hardly any backlash from the fuel cell to the reformer: it operates almost in the same way as it did without being connected to a fuel cell. Fig. 15 gives an overview of the equipment used to connect the reformer and the fuel cell (SQUAREPAK PEM single cell from PRAGMA Industries, $5 \mathrm{~cm}^{2}$ active area, single serpentine flow field, external heating, $70^{\circ} \mathrm{C}$ operating temperature).

Fig. 15. Diagram of the reformer and fuel cell test station

There are only two minor consequences in the joint operation. First, the pressure at the hydrogen outlet of the reformer increases due to the pressure drops along the hydrogen line and auxiliary devices that supply the fuel cell. This pressure drop has been estimated to be only 200 mbar, hence it has a slight reduction on the separation efficiency of the reformer. This negative effect on the hydrogen yield can be compensated by increasing the waste gas pressure. Second, in the test set up, the hydrogen lines and the humidifier introduce an additional time delay for the hydrogen between the output of the reformer and the input of the fuel cell. To account for the slower response of the whole system a 16 second First Order System has been applied to the current set point of the electronic load in order to slow down the dynamics of the hydrogen demand for the reformer.

Fig. 16(a) shows a test run in which the fuel cell output was ramped up to the maximum power point. The controller followed an algorithm which predicts the maximum power peak ( $F C$ Power prediction) in each time step by measuring the inner resistance of the cell. With this prediction the controller calculates the corresponding current ( $F C$ Current prediction) and the necessary hydrogen set point $F_{\mathrm{H} 2}{ }^{*}$ for the hydrogen production of the reformer in order to generate the necessary hydrogen to supply the fuel cell in the maximum power point.

Fig. 16(b) shows the hydrogen set point $F_{\mathrm{H}^{2}}{ }^{*}$ and the measured hydrogen flow rates. It can be seen that the measurement of the second flow meter $F_{H 2}, m 2$ which is closer to the fuel cell is retarded and smoothed compared to the measurement of the first flow meter $F_{H 2}, m 1$. It also follows rather the step-response of a Second Order System. Since the current demand was only slowed down by a First Order System the stoichiometric ratio (Stoichiometric ratio, m2) calculated from the hydrogen measurement of the second meter decreases to values below 1 at the beginning of the test run. If the fuel cell would be located right after the reformer, the upper stoichiometric ratio curve of the first meter (Stoichiometric ratio, m1) - not going below a ratio of 1.4 - would apply [6]. 
Fig. 16. Reformer and fuel cell operation: (a) electrical values of the fuel cell, (b) hydrogen supply values of the fuel cell

Shorter tubes between the reformer and the fuel cell would also decrease their pressure drop. As a general rule, devices with pressure drops from the membrane separation process to the fuel cell need to be omitted. This could be done by using low pressure drop check valves and by taking advantage of the pressure drop of the fuel cell channels to measure the flow rate, allowing to omit the flow meter and also the water trap, which was only used to protect the flow meter. Fuel cells, which are humidified by using only the produced water inside the cell, would make separate humidification of the hydrogen unnecessary.

Even if the reformer is much faster with the proposed control than with a standard PID-controller, it will be still slower than a fuel cell subjected to strong dynamic electrical loads. An electric buffer like a battery or a supercapacitor bank with the capacity to store the electrical energy necessary for several seconds of operation would solve this time mismatch problem. For the use in electric vehicles, fuel cells are always combined with electric buffers. Highly dynamic load changes are mainly supplied by the electric buffer reducing the load change for the fuel cell. In such an application the response time of the presented controlled reformer is sufficient.

\section{CONCLUSIONS}

The static and dynamic characteristics of an ethanol reformer with a low temperature catalytic reformation and membrane separation have been thoroughly studied and the measurement results obtained are highly reproducible. The high purity of the produced hydrogen makes this ethanol reformer compatible with its use to supply hydrogen to a PEM fuel cell. An efficient controller has been proposed and implemented permitting the reduction of the response time of the reformer by a factor of 7 down to 8 seconds by acting simultaneously on the fuel flow rate and pressure. If the hydrogen lines that connect the reformer and the fuel cell are kept short and a small electrical energy buffer is added to the output of the fuel cell, the reformer can be conveniently matched to the dynamic capabilities of fuel cells.

\section{NOMENCLATURE}

\begin{tabular}{l|l|l}
\multicolumn{1}{c|}{ Symbol } & \multicolumn{1}{|c}{ Used in } & \multicolumn{1}{c}{ Description } \\
\hline$\Delta H$ & Eq. 1 to 4 & enthalpy \\
$Q$ & Eq. 5 & Mass flow of membrane separation \\
$P_{e}$ & Eq. 5 & Factor of the Sievert's law \\
$\delta$ & Eq. 5 & Thickness of the metal membrane
\end{tabular}




\begin{tabular}{|c|c|c|}
\hline$A$ & Eq. 5 & Surface area of the metal membrane \\
\hline$P_{W G}$ & $\begin{array}{l}\text { Eq. 5, Fig. 6, Fig. 11, } \\
\text { Fig. 12, Fig. } 13\end{array}$ & Pressure of reformed gas and waste gas \\
\hline$P_{\text {H2_pure }}$ & Eq. 5 & Pressure at pure hydrogen outlet \\
\hline$P^{*}$ & Fig. 12 & Pressure set point \\
\hline$P_{P G F}$ & Fig. 12 & Pressure set point after the pressure control guide filter \\
\hline$\Delta P$ & Fig. 13 & Pressure difference for pressure release \\
\hline$F_{\mathrm{H} 2}$ & Eq. 6, Fig. 13 & Hydrogen flow rate \\
\hline$F_{H 2}{ }^{*}$ & Fig. 13 & Hydrogen flow rate set point \\
\hline$F_{H 2 \_F F}$ & Fig. 11, Fig. 13 & Hydrogen flow rate due to fuel flow rate control \\
\hline$F_{H 2 \_} P$ & Fig. 12, Fig. 13 & Hydrogen flow rate due to pressure control \\
\hline$e_{H 2}$ & Fig. 13 & Hydrogen flow rate control error \\
\hline$\Delta F_{\mathrm{H} 2}$ & Eq. 6 & $\begin{array}{l}\text { Difference in hydrogen flow rate between one operating } \\
\text { condition to another }\end{array}$ \\
\hline$F_{\text {Fuel }}$ & $\begin{array}{l}\text { Fig. 3, Fig. 11, Fig. 12, } \\
\text { Fig. } 13\end{array}$ & Fuel flow rate \\
\hline$F_{\text {Fuel_FF }}$ & Fig. 13 & Fuel flow rate due to feed forward control action \\
\hline$F_{\text {Fuel_P }}$ & Fig. 13 & Fuel flow rate due to proportional control action \\
\hline$F_{\text {Fuel_I }}$ & Fig. 13 & Fuel flow rate due to integral control action \\
\hline$F F$ & & Feed forward control \\
\hline$\tau_{F F}$ & Fig. 11 & $\begin{array}{l}\text { Time constant of the reformer to adjust between two operating } \\
\text { conditions }\end{array}$ \\
\hline$P_{\mathrm{H} 2}$ & Fig. 6 & Hydrogen partial pressure \\
\hline$F_{W G}$ & $\begin{array}{l}\text { Fig. 3, Fig. 6, Fig. 12, } \\
\text { Fig. } 13\end{array}$ & Waste gas flow rate \\
\hline Trel & Fig. 7 & Time at pressure release \\
\hline Tdead & Fig. 7 & Dead time for pressure release \\
\hline Tpeak, $T_{P k}$ & Fig. 7, Fig. 8, Fig. 13 & Time at which the peak value of hydrogen occurs \\
\hline Tdown & Fig. 7 & Time at which the effect of a pressure release is over \\
\hline$H 2_{P k}$ & Fig. 7, Fig. 8, Fig. 13 & Hydrogen peak value after pressure release \\
\hline$P 1$ & Fig. 7 & Pressure before pressure release \\
\hline$P 2$ & Fig. 7 & Pressure after pressure release \\
\hline
\end{tabular}




\begin{tabular}{|c|c|c|}
\hline H2stat1 & Fig. 7 & Static hydrogen flow rate at pressure P1 \\
\hline H2stat2 & Fig. 7 & Static hydrogen flow rate at pressure P2 \\
\hline$T_{F F}$ & Fig. 11, Fig. 13 & Time constant of the fuel pump \\
\hline$T_{P T 1}, T_{P T 2}$ & Fig. 12 & $\begin{array}{l}\text { Time constants of the Second Order Element to shape the } \\
\text { modeled pressure release peak }\end{array}$ \\
\hline$T_{\text {valve }}$ & Fig. 12 & Time constant of the pressure control valve \\
\hline$T_{P G F}$ & Fig. 12 & Time constant of the pressure control guide filter \\
\hline$T t$ & Fig. 13 & Dead time for smith predictor in proportional control part \\
\hline$K_{P}$ & Fig. 13, Table 1 & Amplification factor of proportional control part \\
\hline$K_{l}$ & Fig. 13, Table 1 & Amplification factor of integral control part \\
\hline $\mathrm{K}_{3} \mathrm{H}_{2} \mathrm{FF}$ & Fig. 13 & $\begin{array}{l}\text { Amplification factor of the reformer system, } \\
\text { equals hydrogen flow rate }[\mathrm{ml} / \mathrm{min}] / \text { fuel flow rate }[\mathrm{ul} / \mathrm{min}]\end{array}$ \\
\hline$F_{\text {valve }}$ & Fig. 12 & Flow rate before the waste gas pressure control valve \\
\hline$F_{\text {out }}$ & Fig. 12 & Flow rate leaving the waste gas pressure control valve \\
\hline Volume & Fig. 12 & Volume of the reformer core \\
\hline
\end{tabular}

\section{PARAMETERS}

\begin{tabular}{|c|c|c|}
\hline Figure & Parameter & Equation, Value \\
\hline \multirow[t]{4}{*}{ Fig. 5} & $\tau_{F F}\left(P_{W G}, F_{H 2 \_F F}\right)$ & $=\left(K 1 \tau_{F F}+K 2 \tau_{F F} \cdot P_{W G}+K 3 \tau_{F F} \cdot F_{H 2_{F} F F}\right)^{-1}$ \\
\hline & $K 1 \tau_{F F}$ & $=41.2 * 10^{-3}$ \\
\hline & $K 2 \tau_{F F}$ & $=-2.75 * 10^{-3}$ \\
\hline & $K 3 \tau_{F F}$ & $=0.802 * 10^{-3}$ \\
\hline \multirow[t]{4}{*}{ Fig. 8} & $T_{P k}\left(P_{W G}, F_{\text {Fuel }}\right)$ & $=\left(K 1_{T P k}+K 2_{T P k} \cdot P_{W G}+K 3_{T P k} \cdot F_{F u e l}\right)$ \\
\hline & $K 1_{T P k}$ & $=7.20$ \\
\hline & $K 2_{T P k}$ & $=-0.190$ \\
\hline & $K 3_{T P k}$ & $=-5.67 * 10^{-3}$ \\
\hline \multirow[t]{5}{*}{ Fig. 8} & $H 2_{P k}\left(P_{W G}, F_{\text {Fuel }}\right)$ & $=\left(K 1_{H 2 P k}+K 2_{H 2 P k} \cdot P_{W G}+K 3_{H 2 P k} \cdot F_{F u e l}+\right.$ \\
\hline & & $\left.K 4_{H 2 P k} \cdot P_{W G}{ }^{2}+K 5_{H 2 P k} \cdot P_{W G} \cdot F_{F u e l}+K 6_{H 2 P k} \cdot F_{F u e l}{ }^{2}\right)^{-1}$ \\
\hline & $K 1_{\text {H2Pk }}$ & $=0.240$ \\
\hline & $K 2_{H 2 P k}$ & $=-29.1 * 10^{-3}$ \\
\hline & $K 3_{H 2 P k}$ & $=-0.213 * 10^{-3}$ \\
\hline
\end{tabular}




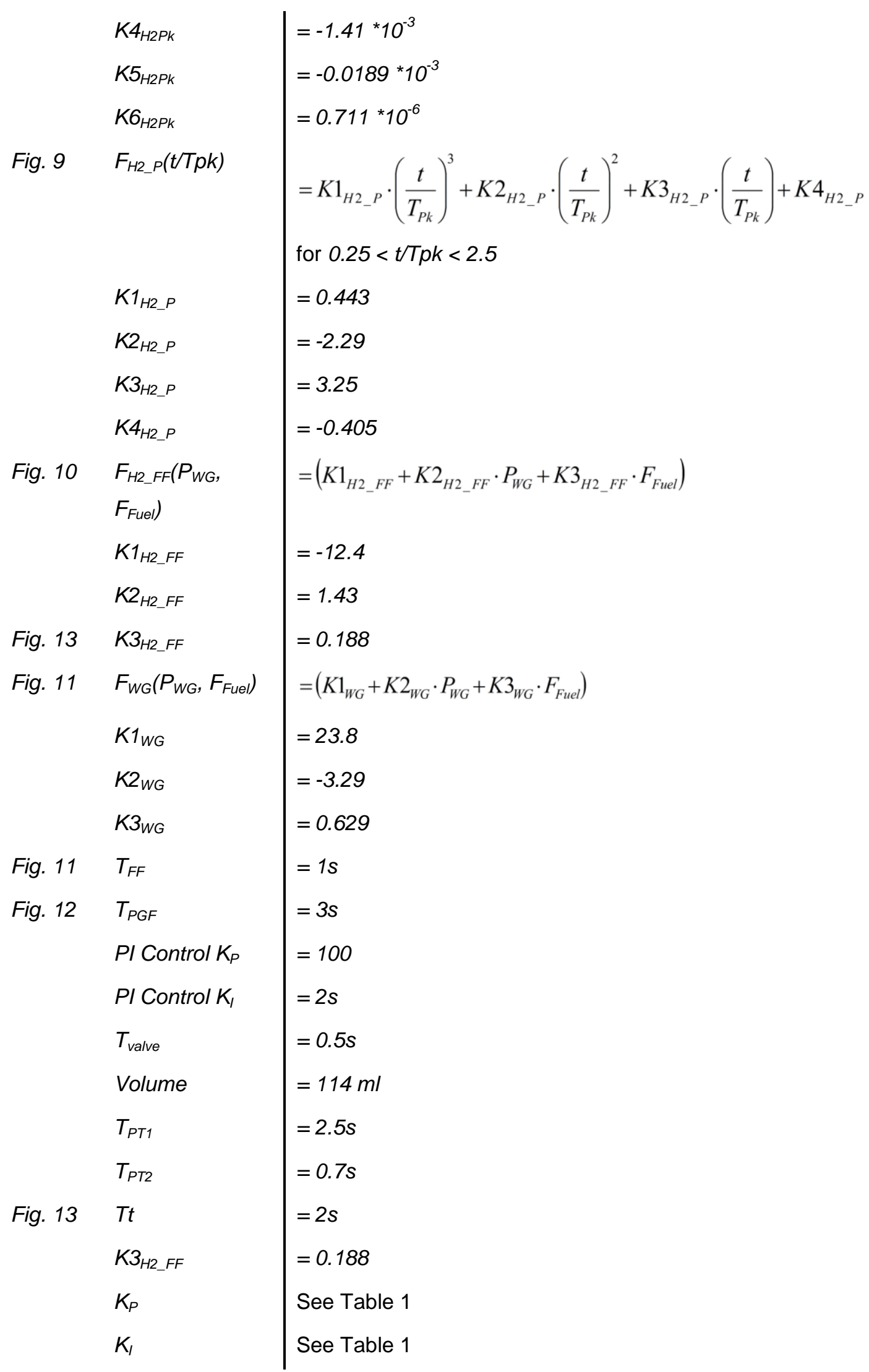




\section{ACKNOWLEDGEMENTS}

The experimental tests were performed at the laboratories of the Institut de Tècniques Energètiques (UPC) and of the Institut de Robòtica I Informàtica Industrial (UPC/CSIC). The authors acknowledge financial support from Generalitat de Catalunya (VALTEC-08-2-0021) and MICINN (CTQ2009-12520, DPI2010-15274 and DPI2011-25649). J.L. is grateful to ICREA Academia program.

\section{REFERENCES}

[1] Llorca, J. Microreactors for the Generation of Hydrogen from Ethanol. Handbook of Sustainable Energy, chapter 22, pp. 693-699. Ed. W. H. Lee and V. G. Cho. Nova Publishers, 2010.

[2] López, E., Divins, N.J., Llorca, J. Hydrogen Production from Ethanol over $\mathrm{PdRh} / \mathrm{CeO}$ with a Metallic Membrane Reactor. Catal. Today, in press.

[3] Idriss, H., Scott, M., Llorca, J., Chan, S.C., Chiu, W., Sheng, P.Y., Yee, A., Blackford, M.A., Pas, S.J., Hill, A.J., Alamgir, F.M., Rettew, R., Petersburg, C., Senanayake, S., Barteau, M.A. A phenomelogical study of the metal-oxide interface: The role of catalysis in hydrogen production from renewable resources. ChemSusChem, 2008, 1, 905-910.

[4] Äström, K. and Hägglund, T., PID Controllers: Theory design and Tuning. ISA 1995.

[5] Skogestad, S. and Postlethwaite, I., Multivariable feedback control. John Wiley and sons, 1996.

[6] Barbir, F., PEM Fuel Cells: Theory and Practice. Elsevier (Sustainable World Series), 2005. 
Tables:

Table 1. Performance of different controllers

\begin{tabular}{|c|c|c|c|c|c|c|c|c|}
\hline$\underset{K_{P}}{P \text {-Controller }}$ & $\underset{K_{l}}{\text { I-Controller }}$ & $\begin{array}{c}\text { Feed- } \\
\text { forward }\end{array}$ & $\begin{array}{l}\text { Smith } \\
\text { Predictor }\end{array}$ & $\begin{array}{l}\text { Pressure } \\
\text { Controller }\end{array}$ & Stability & Damping & Accuracy & $\begin{array}{c}\text { Response } \\
\text { time }\end{array}$ \\
\hline- & - & $\checkmark$ & - & - & $\checkmark$ & $\checkmark$ & - & $\approx 50 \mathrm{~s}$ \\
\hline 5 & $\left(2 \times \tau_{\mathrm{FF}}\right)^{-1}$ & - & - & - & $\checkmark$ & $\checkmark$ & $\checkmark$ & $\approx 60 \mathrm{~s}$ \\
\hline 5 & $\left(2 \times \tau_{\mathrm{FF}}\right)^{-1}$ & $\checkmark$ & - & - & $\checkmark$ & $\checkmark$ & $\checkmark$ & $\approx 12 \mathrm{~s}$ \\
\hline $2 \times \tau_{\mathrm{FF}} \times K_{\mathrm{H}_{2} \mathrm{FF}}$ & $\left(2 \times \tau_{F F F}\right)^{-1}$ & $\checkmark$ & $\checkmark$ & - & $\checkmark$ & $\checkmark$ & $\checkmark$ & $\approx 10 \mathrm{~s}$ \\
\hline $2 \times \tau_{\mathrm{FF}} \times \mathrm{K} 3_{\mathrm{H} 2 \mathrm{FF}}$ & $\left(2 \times \tau_{\mathrm{FF}}\right)^{-1}$ & $\checkmark$ & $\checkmark$ & $\checkmark$ & $\checkmark$ & $\checkmark$ & $\checkmark$ & $\approx 8 \mathrm{~s}$ \\
\hline
\end{tabular}




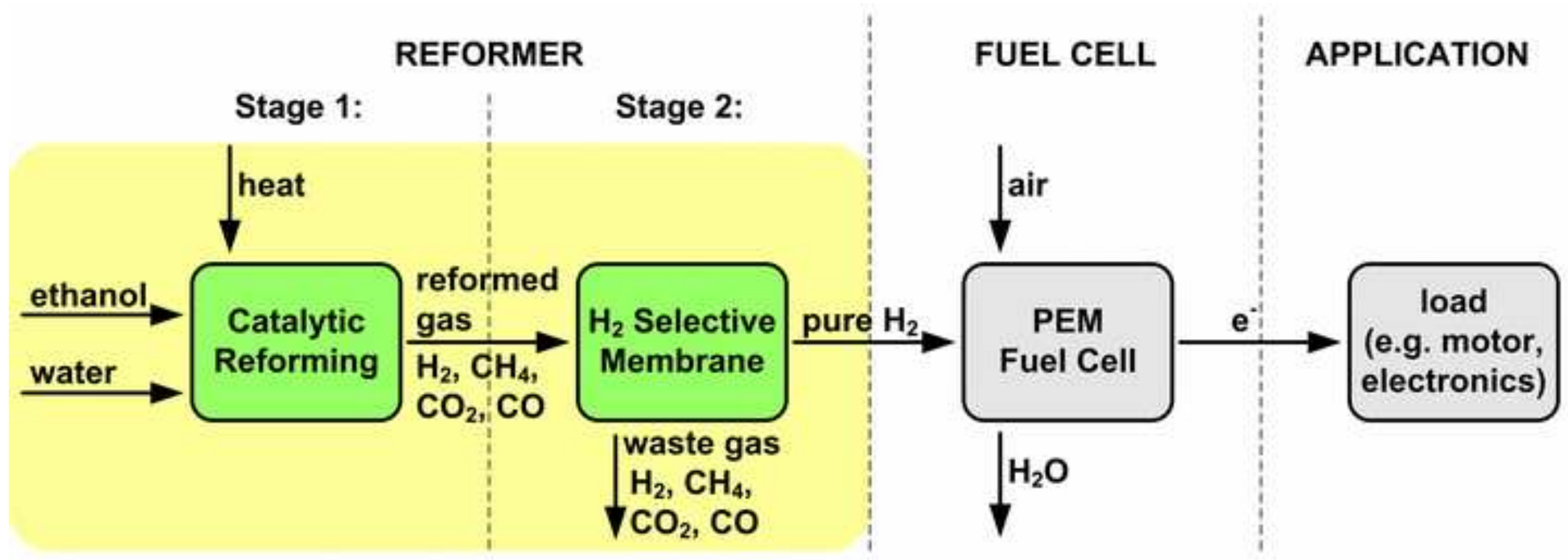



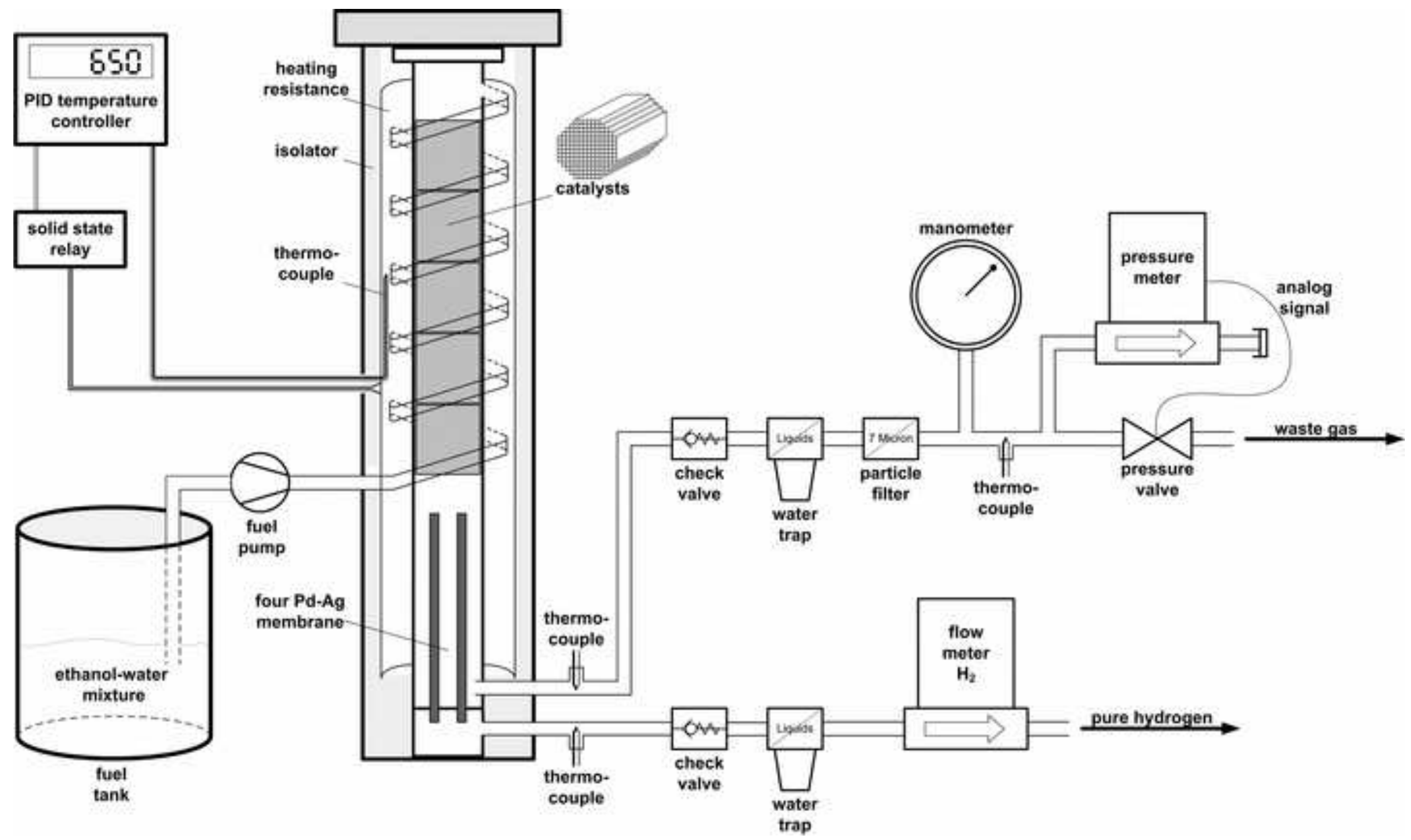


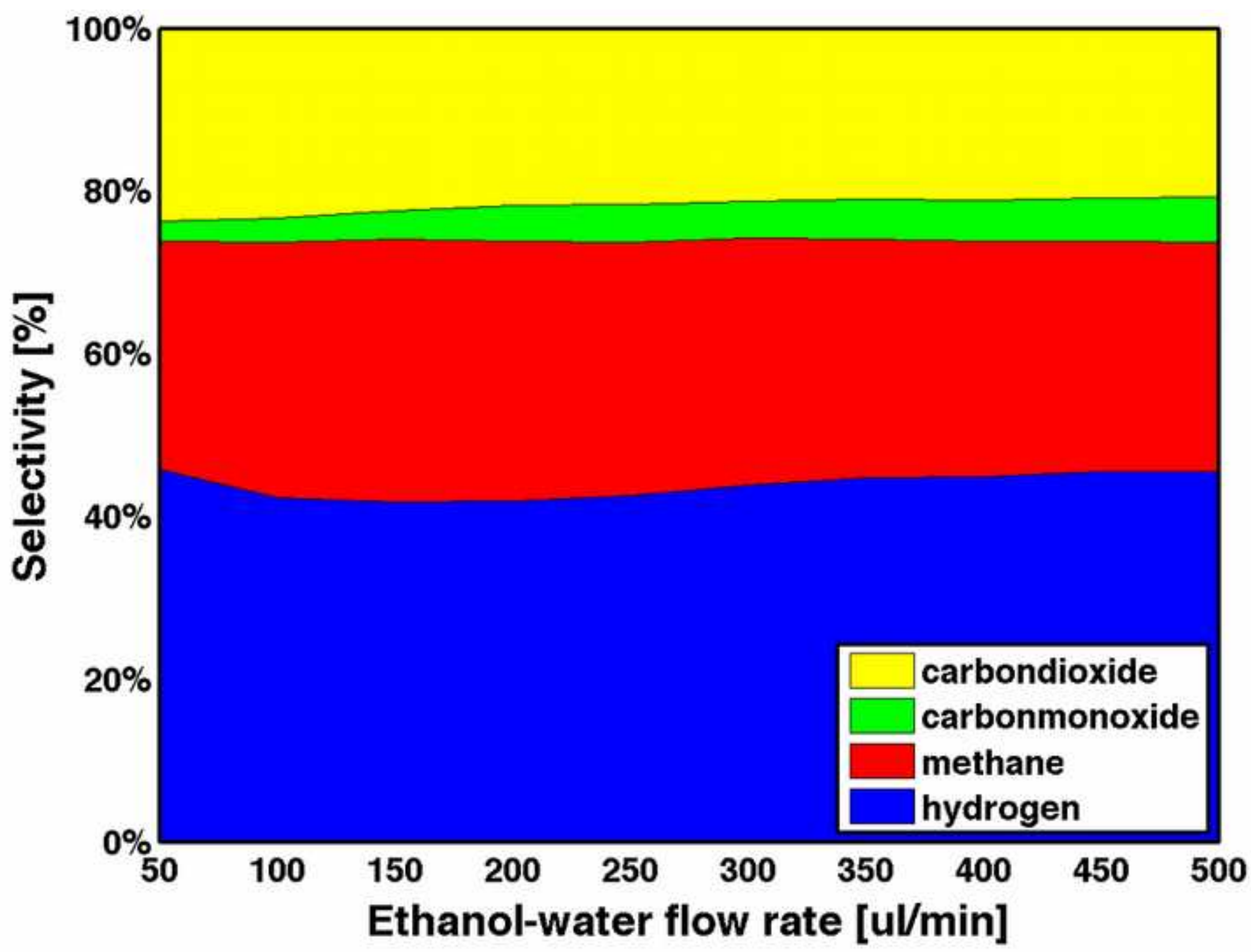



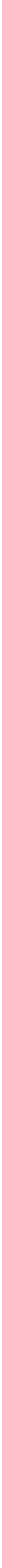

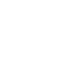

.

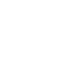

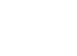

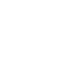
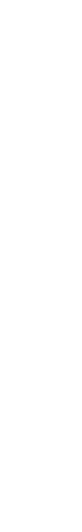

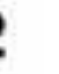

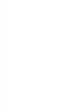




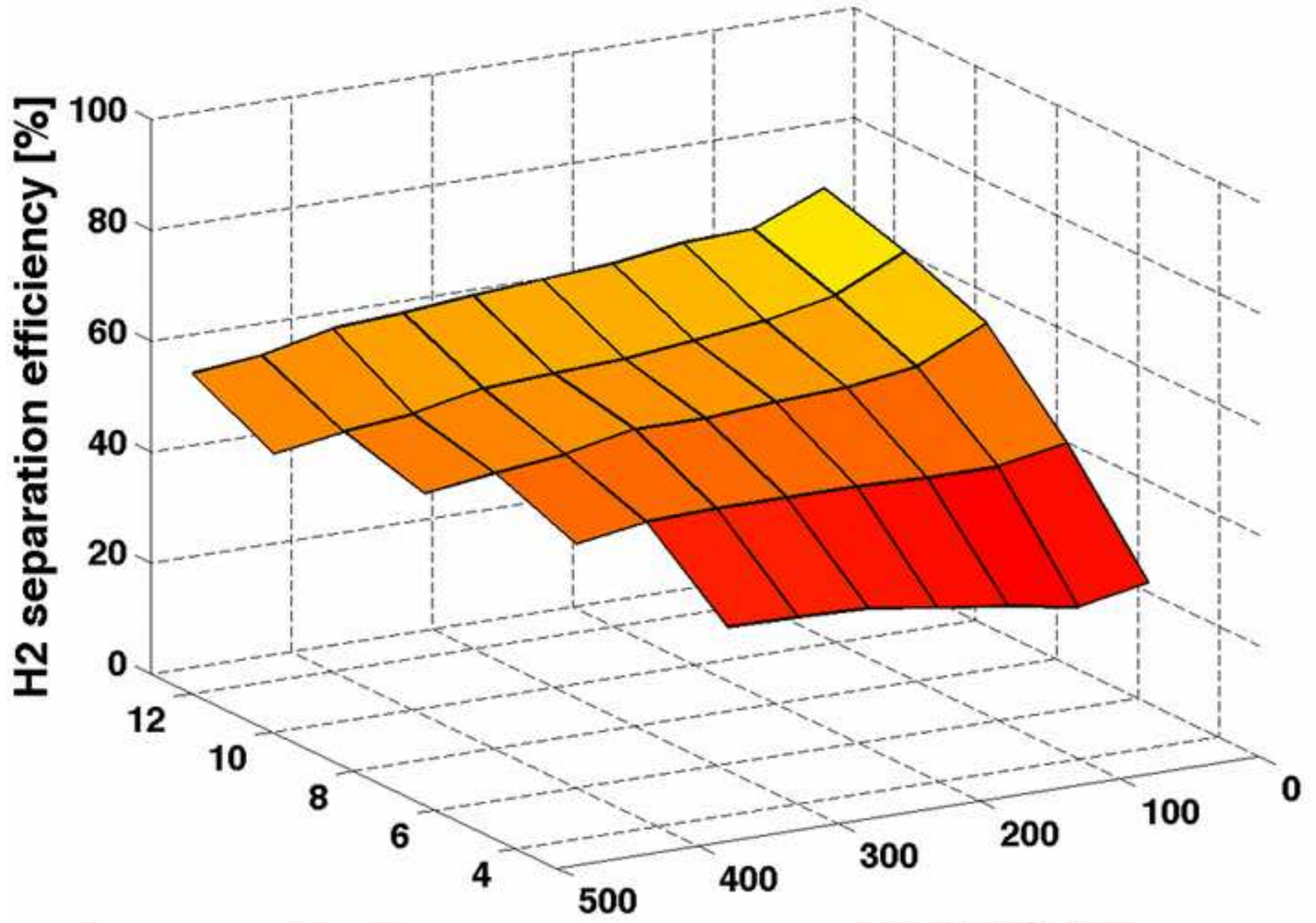

Pressure [bar]

Fuel [ul/min] 


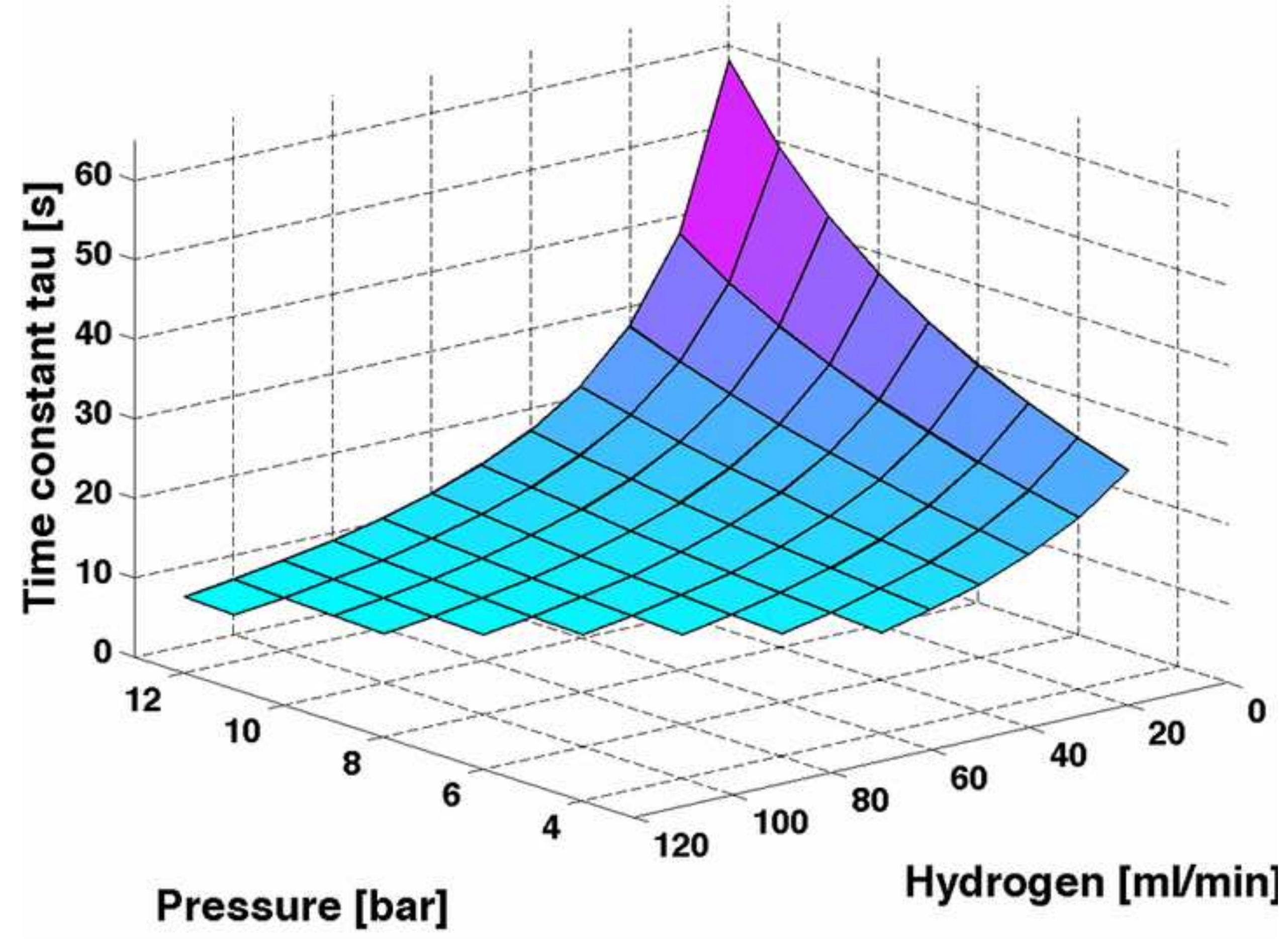




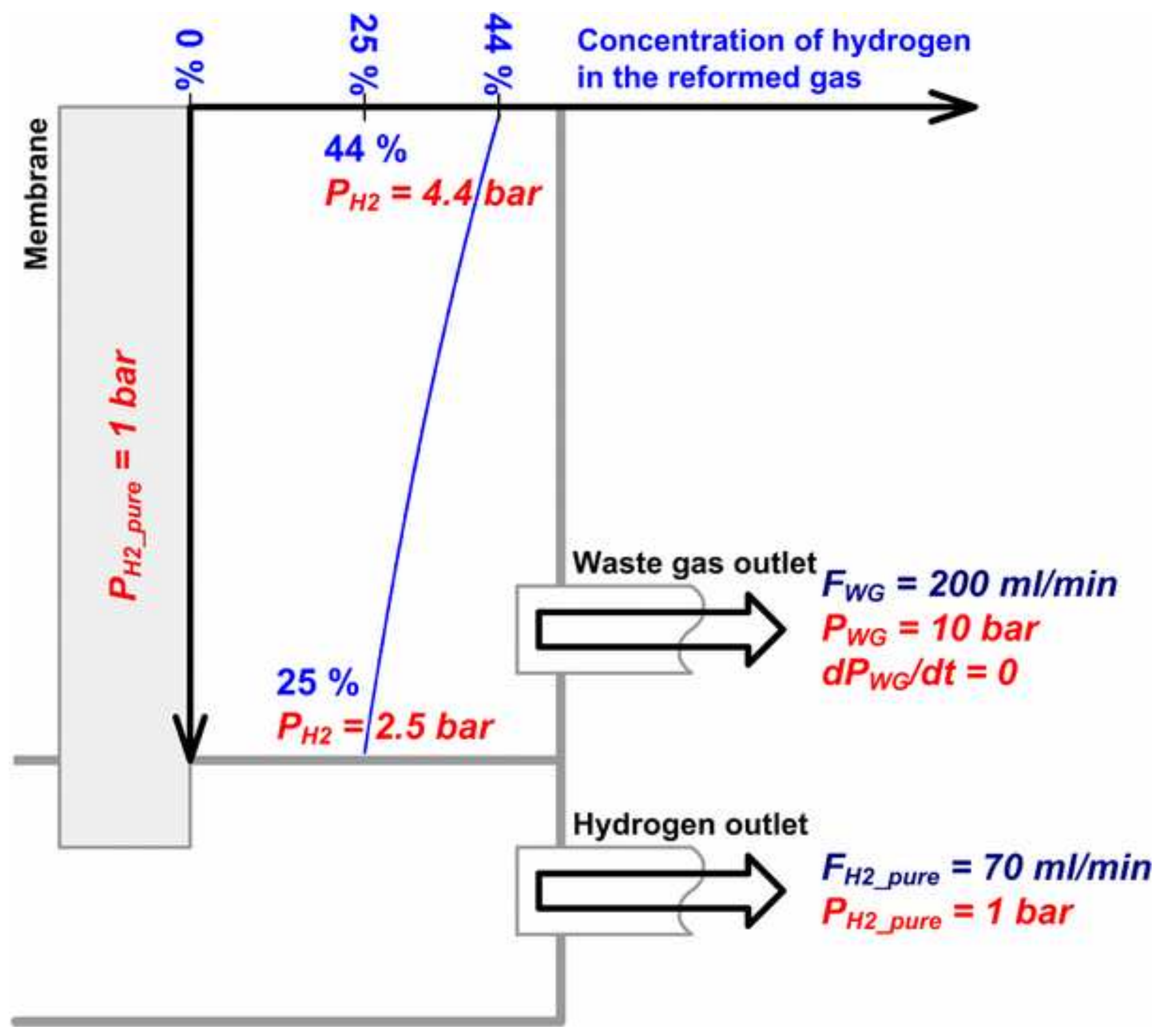




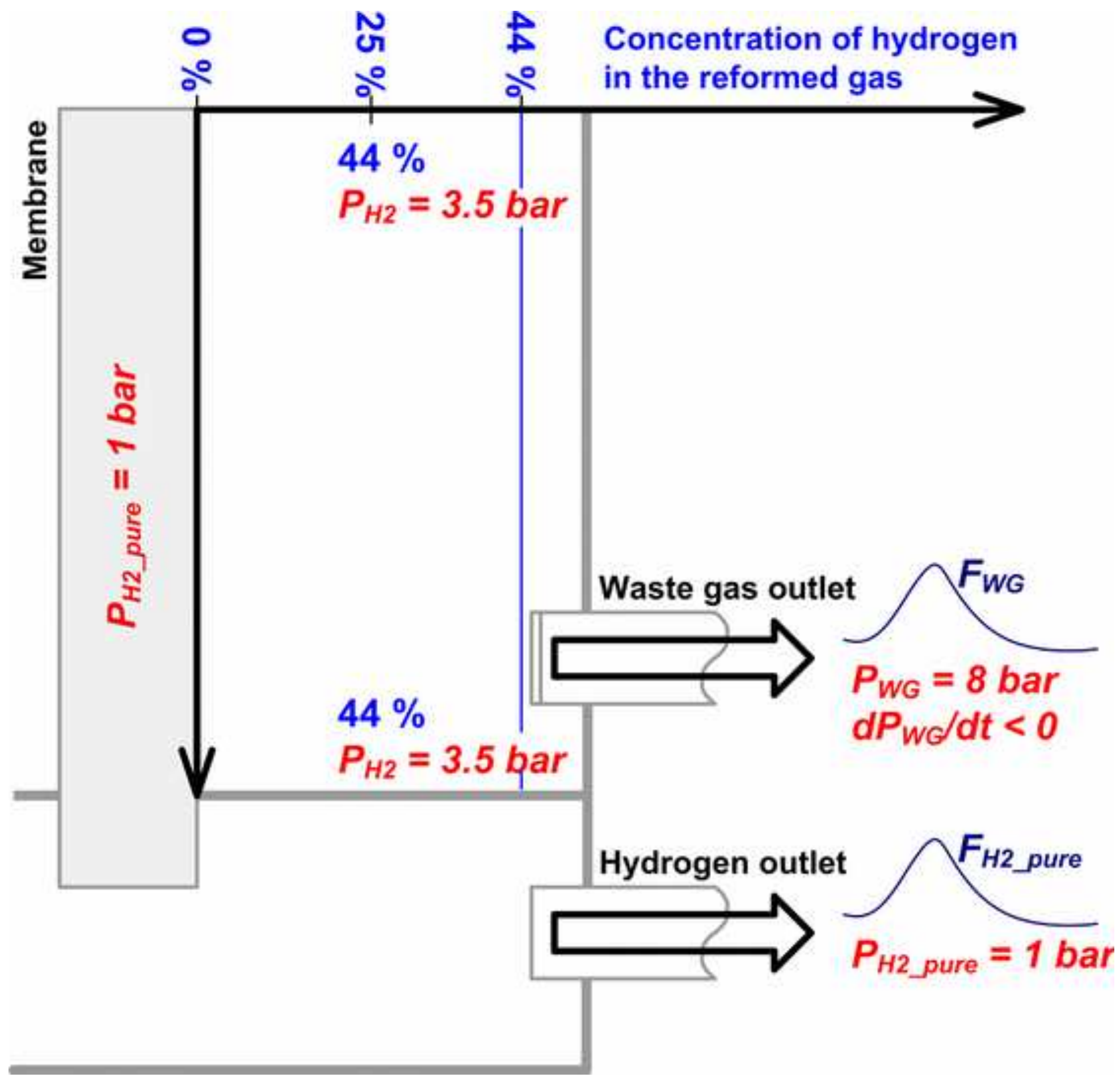




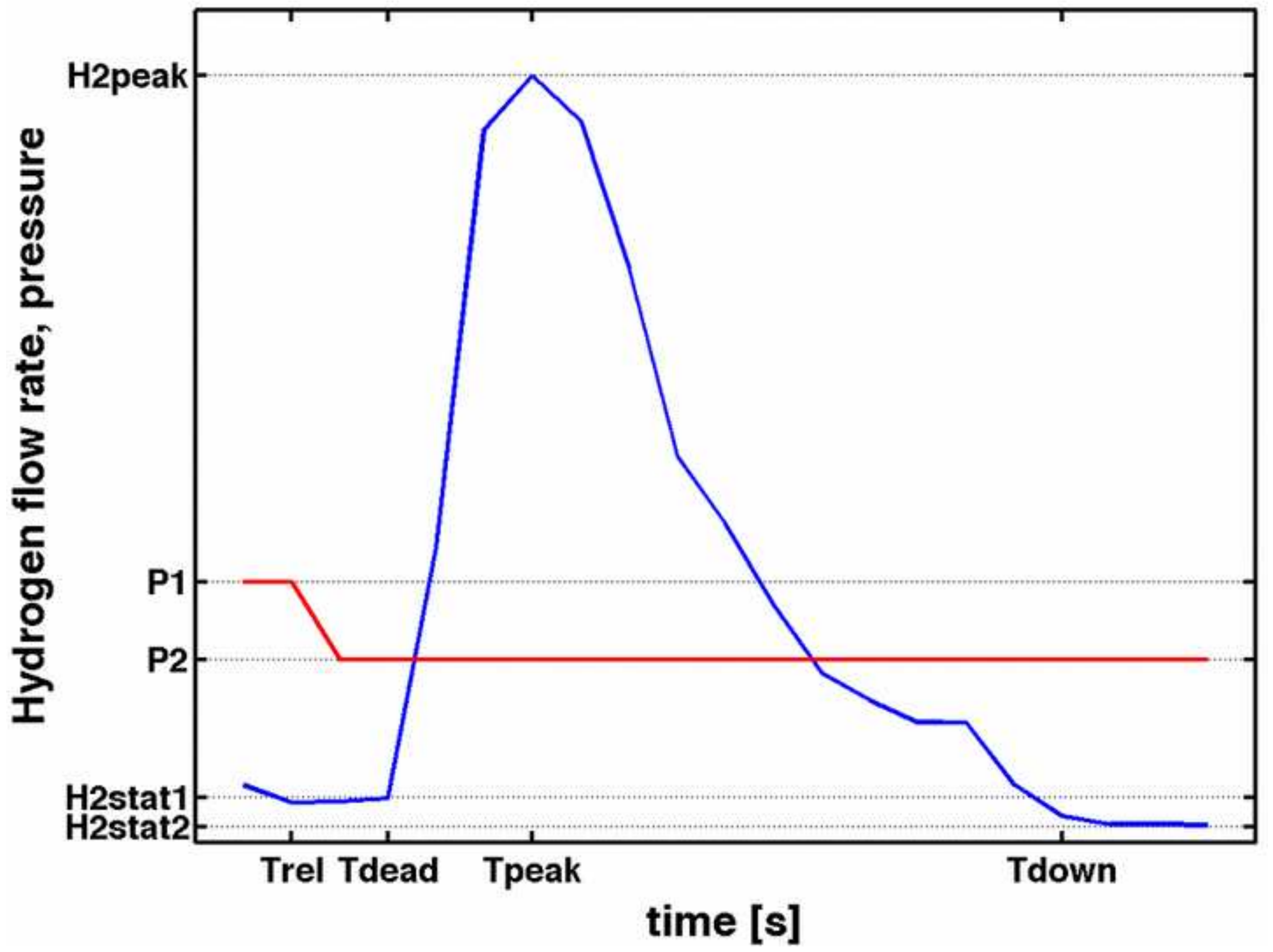




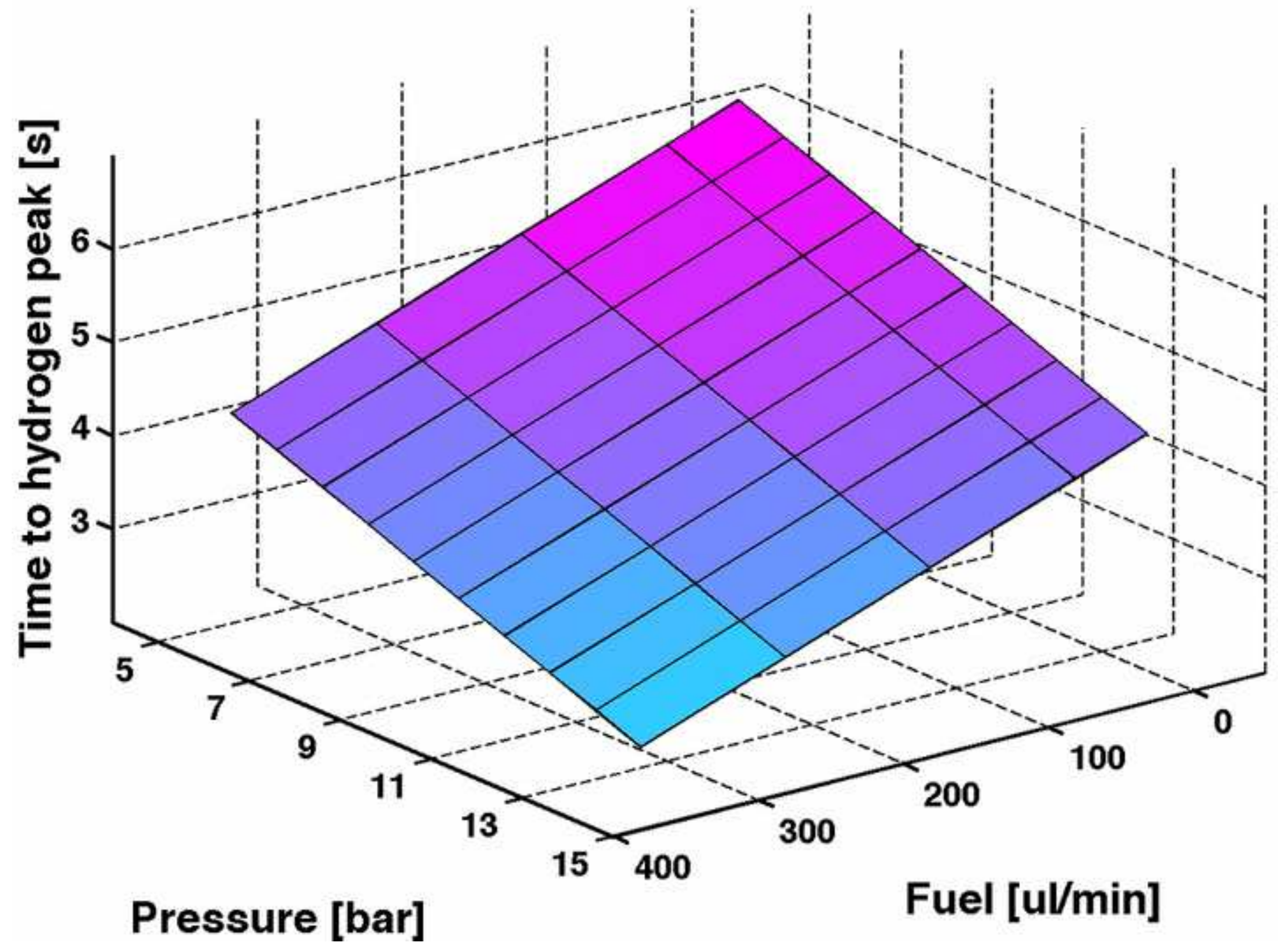




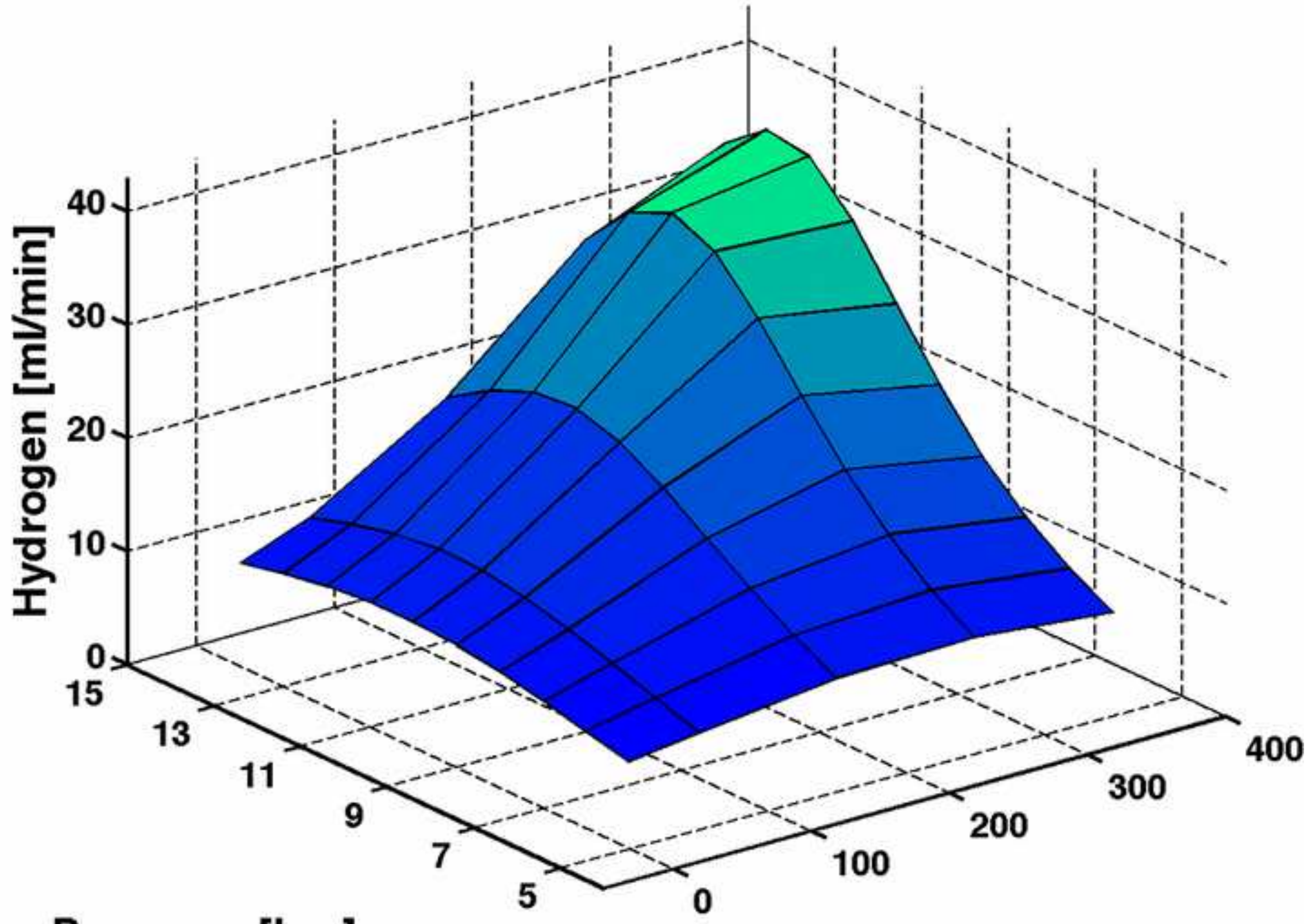

Pressure [bar]

Fuel [ul/min] 


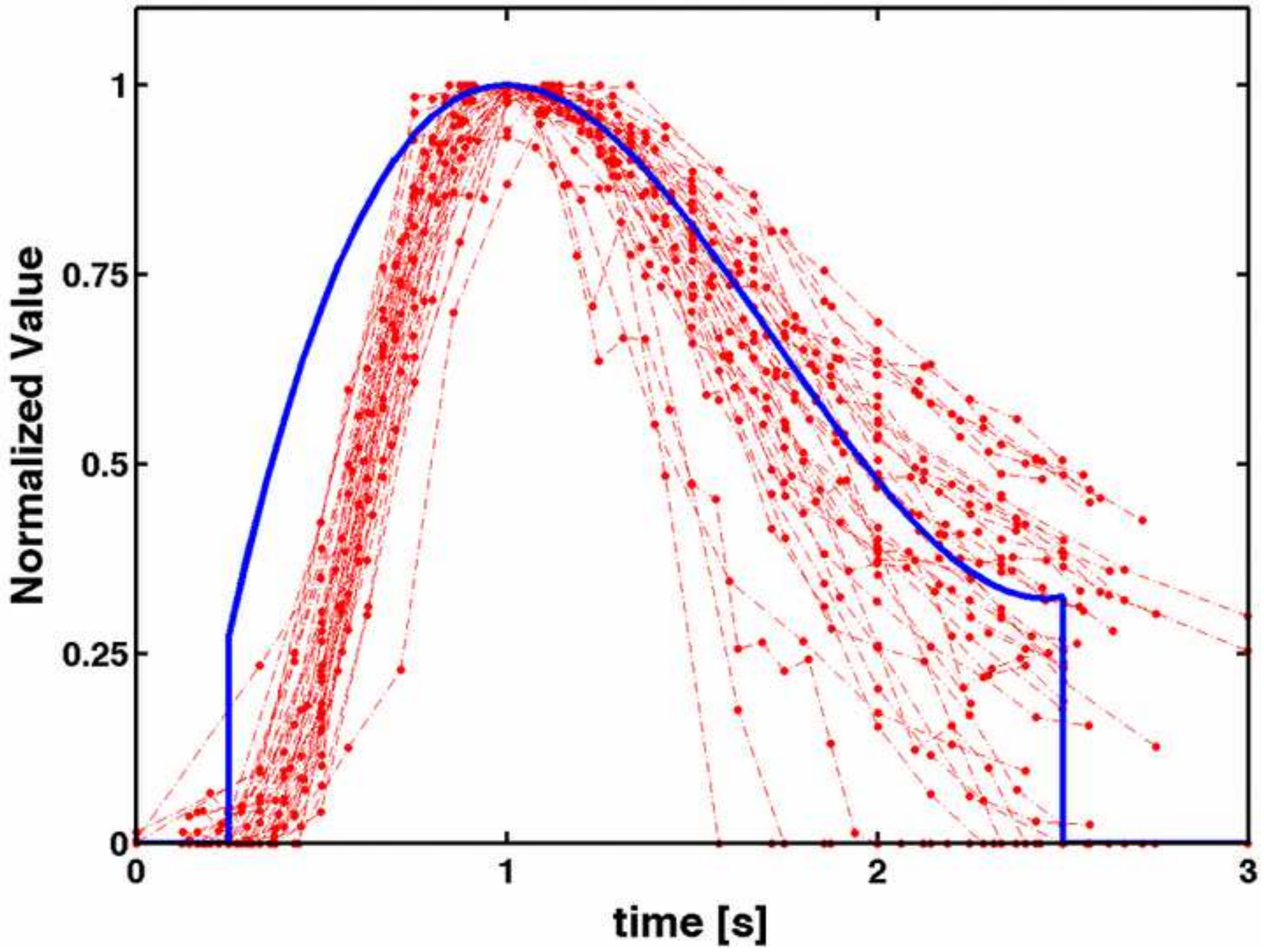




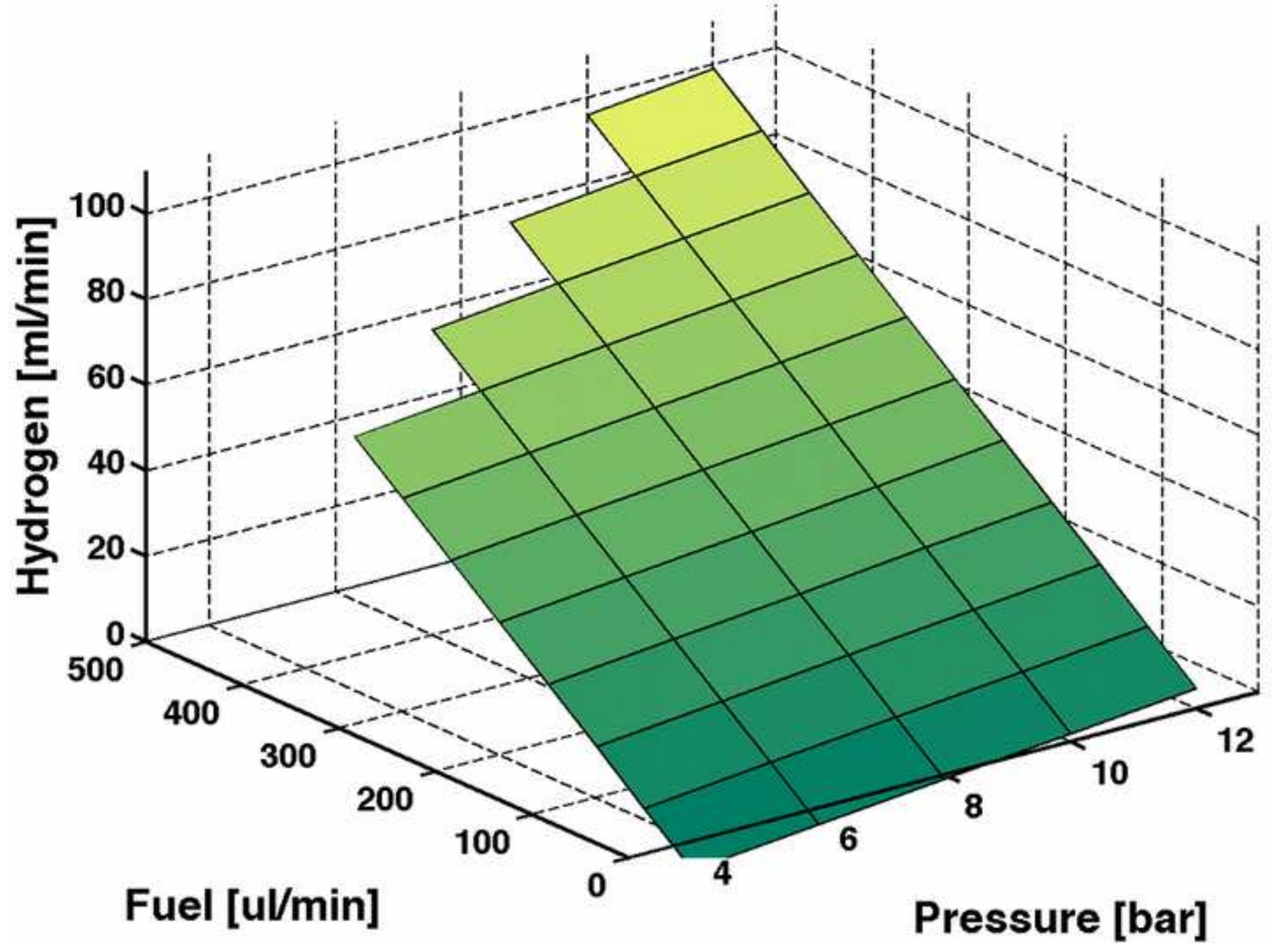




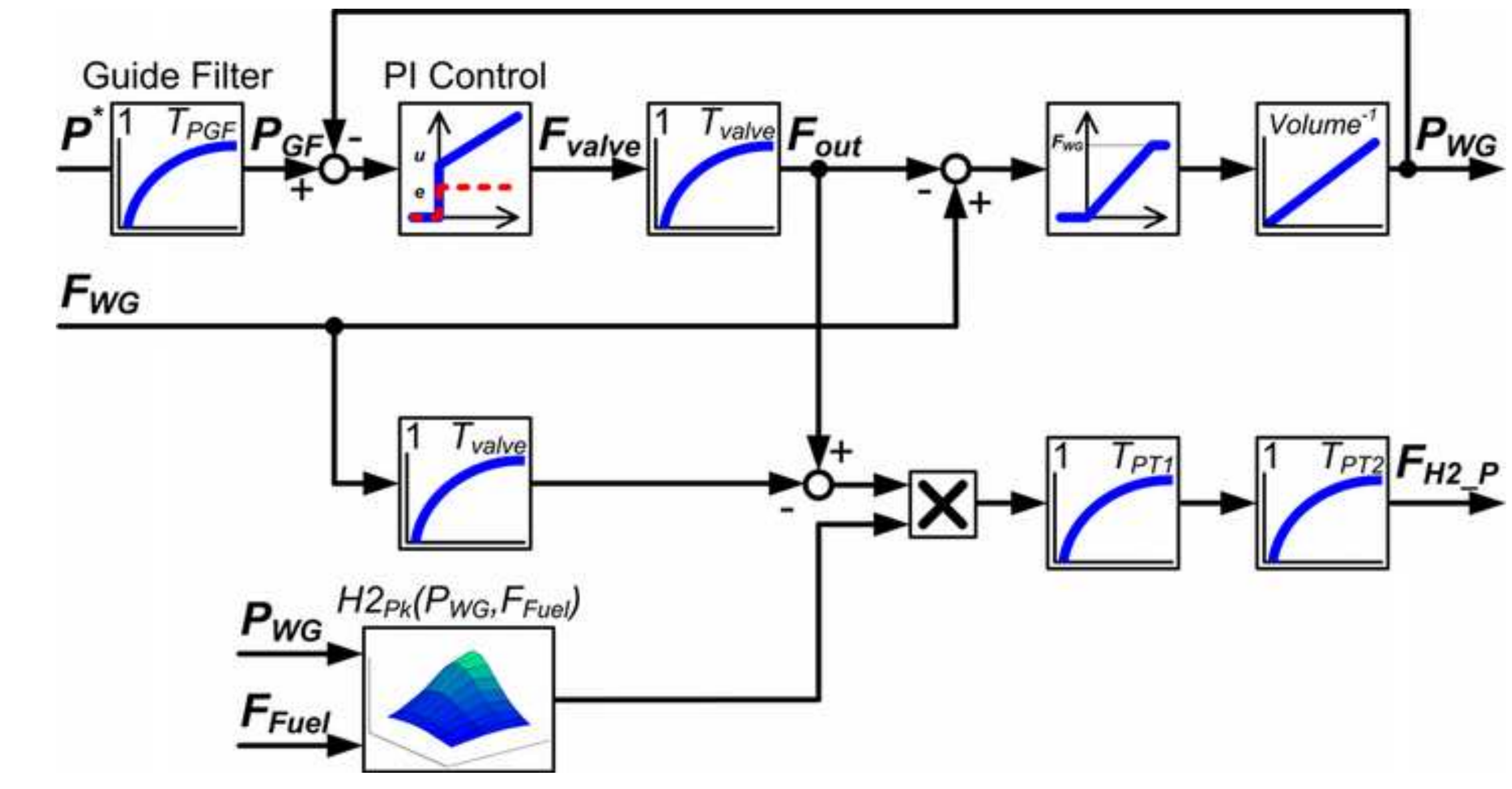

\begin{abstract}
click here to download high resolution image
\end{abstract}


Fig13a Controller action: fuel flow rate

Click here to download high resolution image

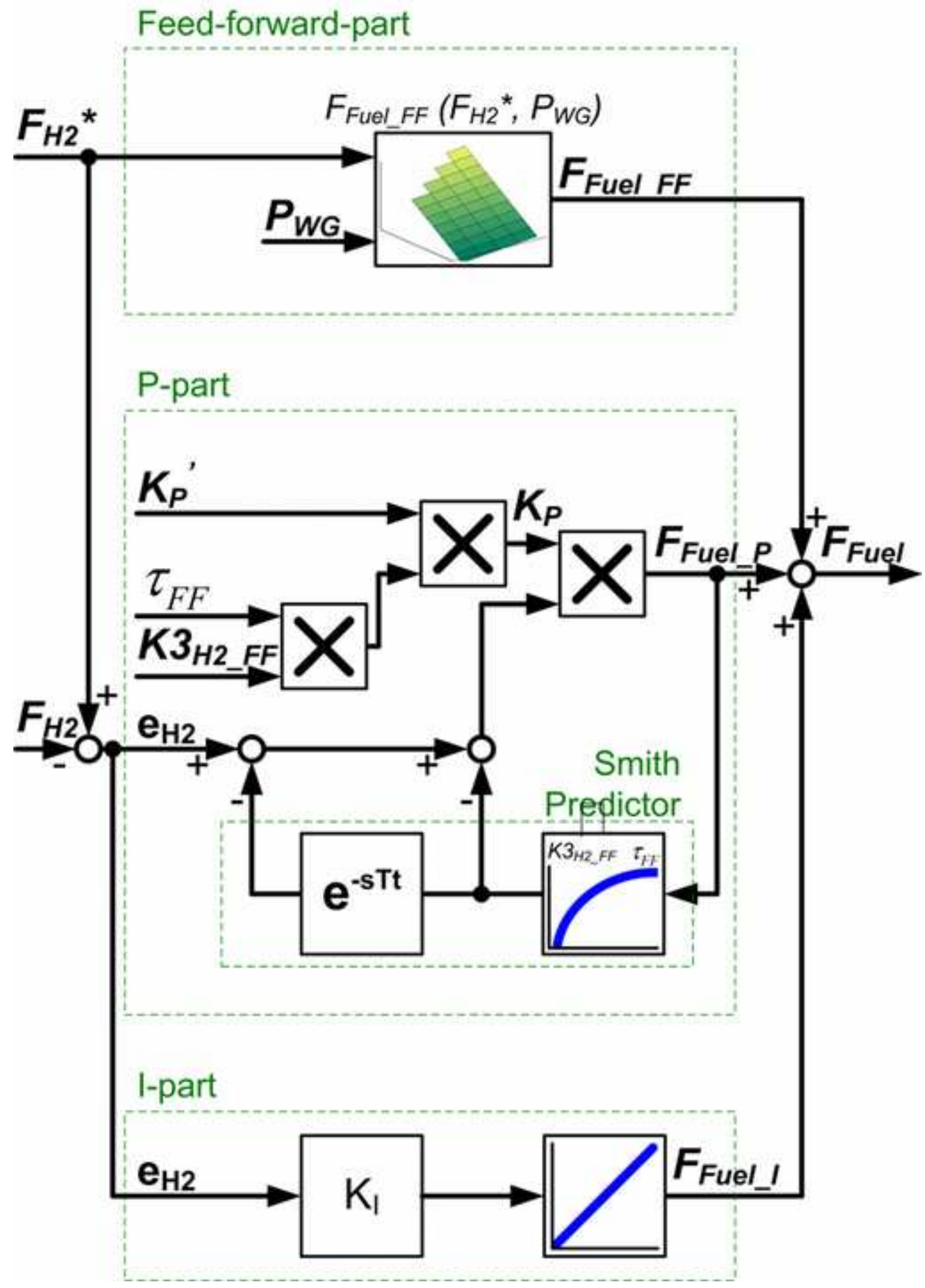




\section{Predictive Control}

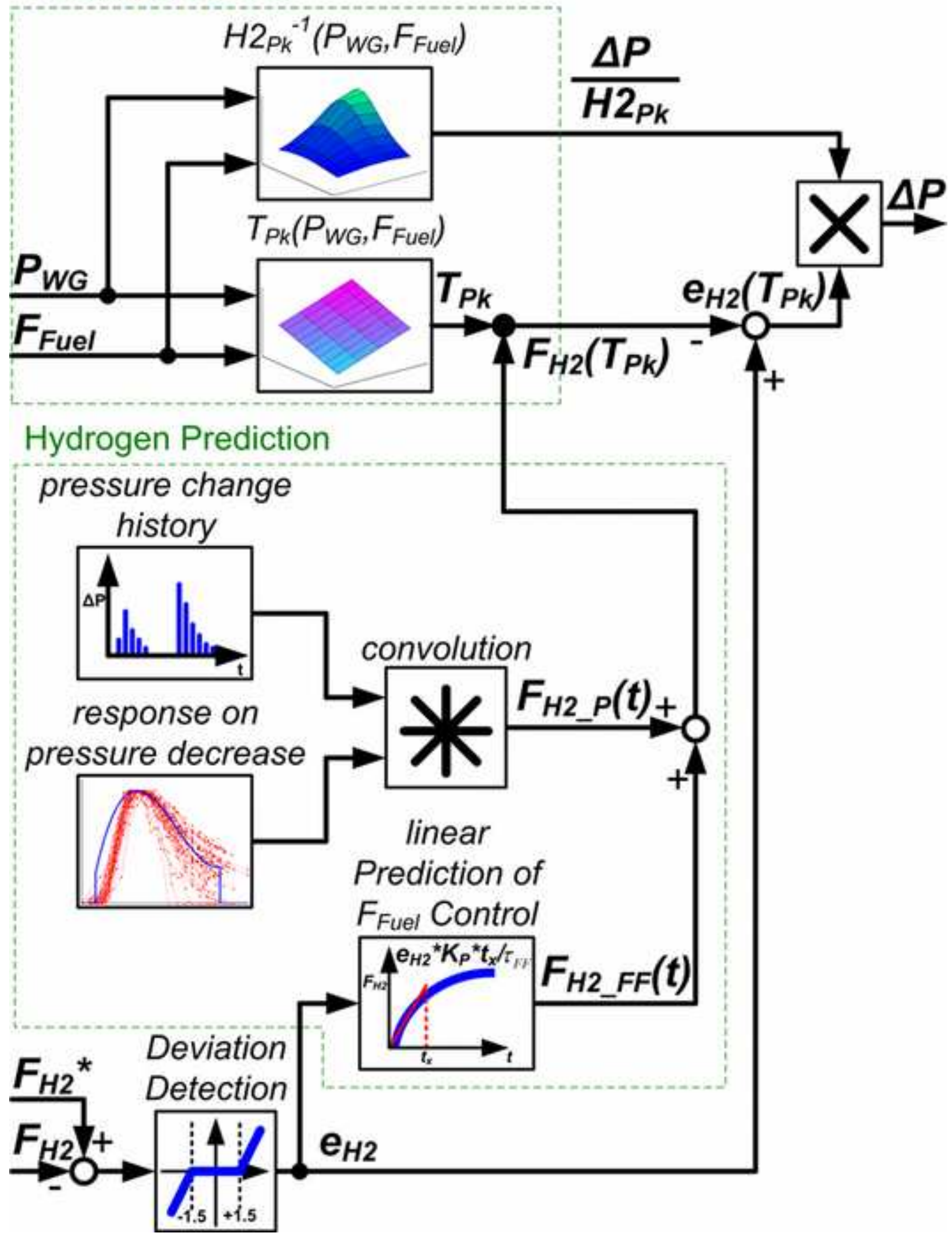



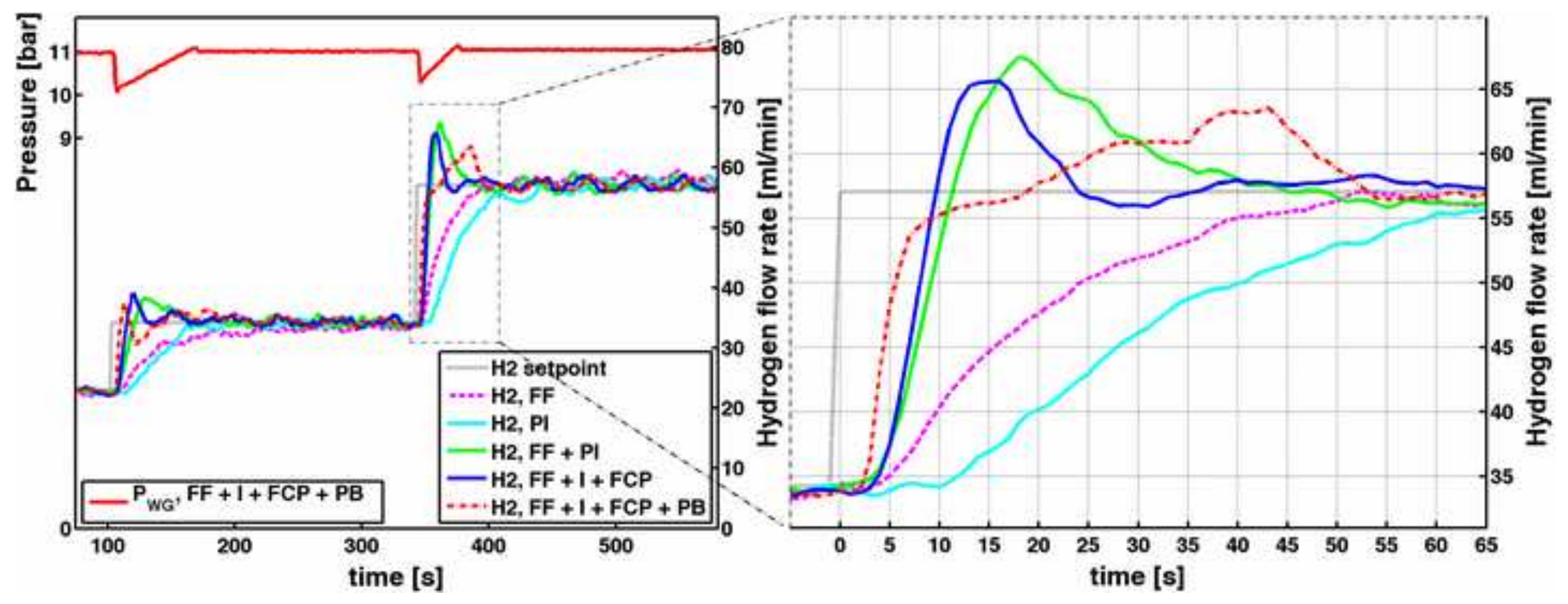


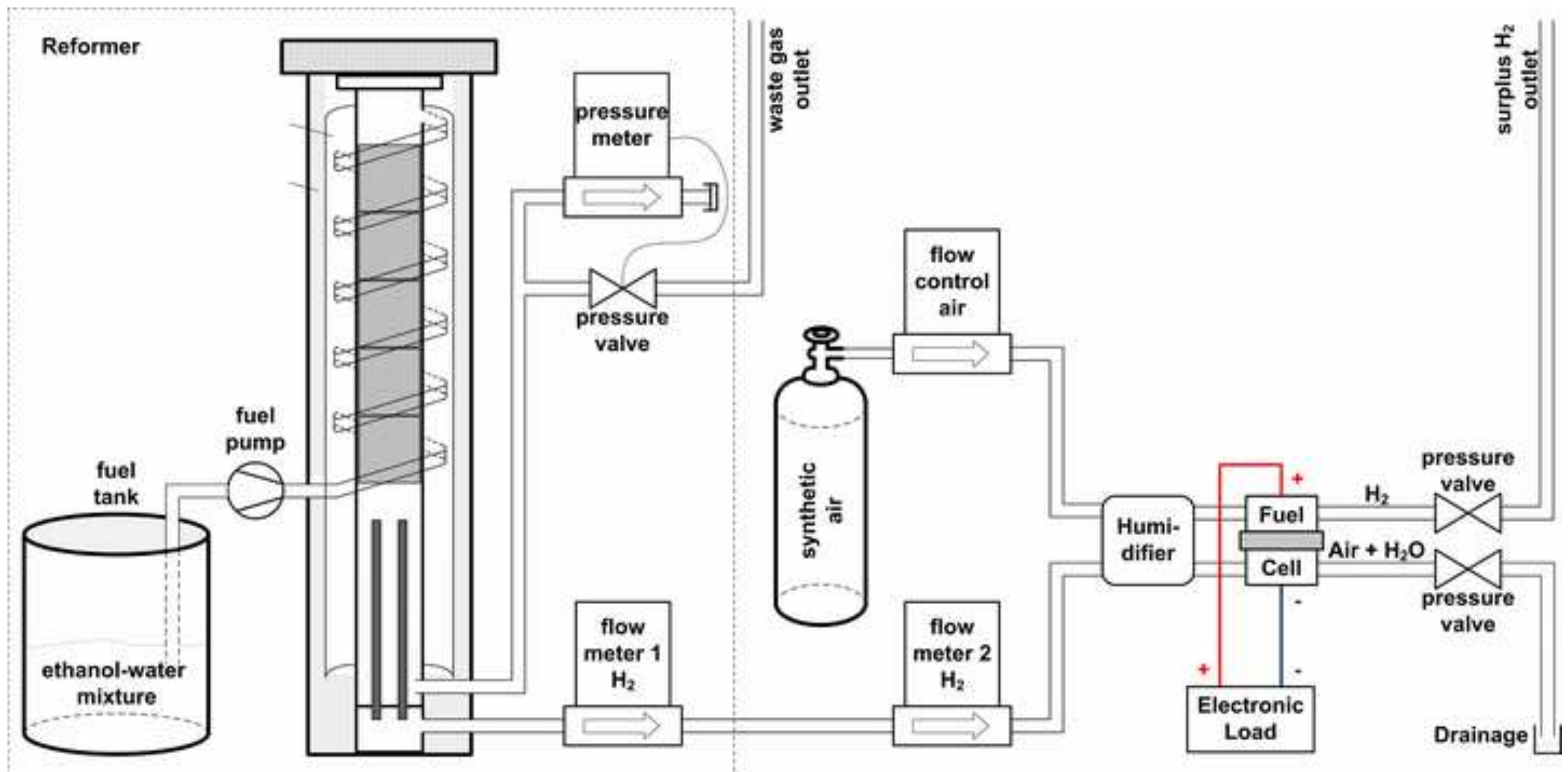




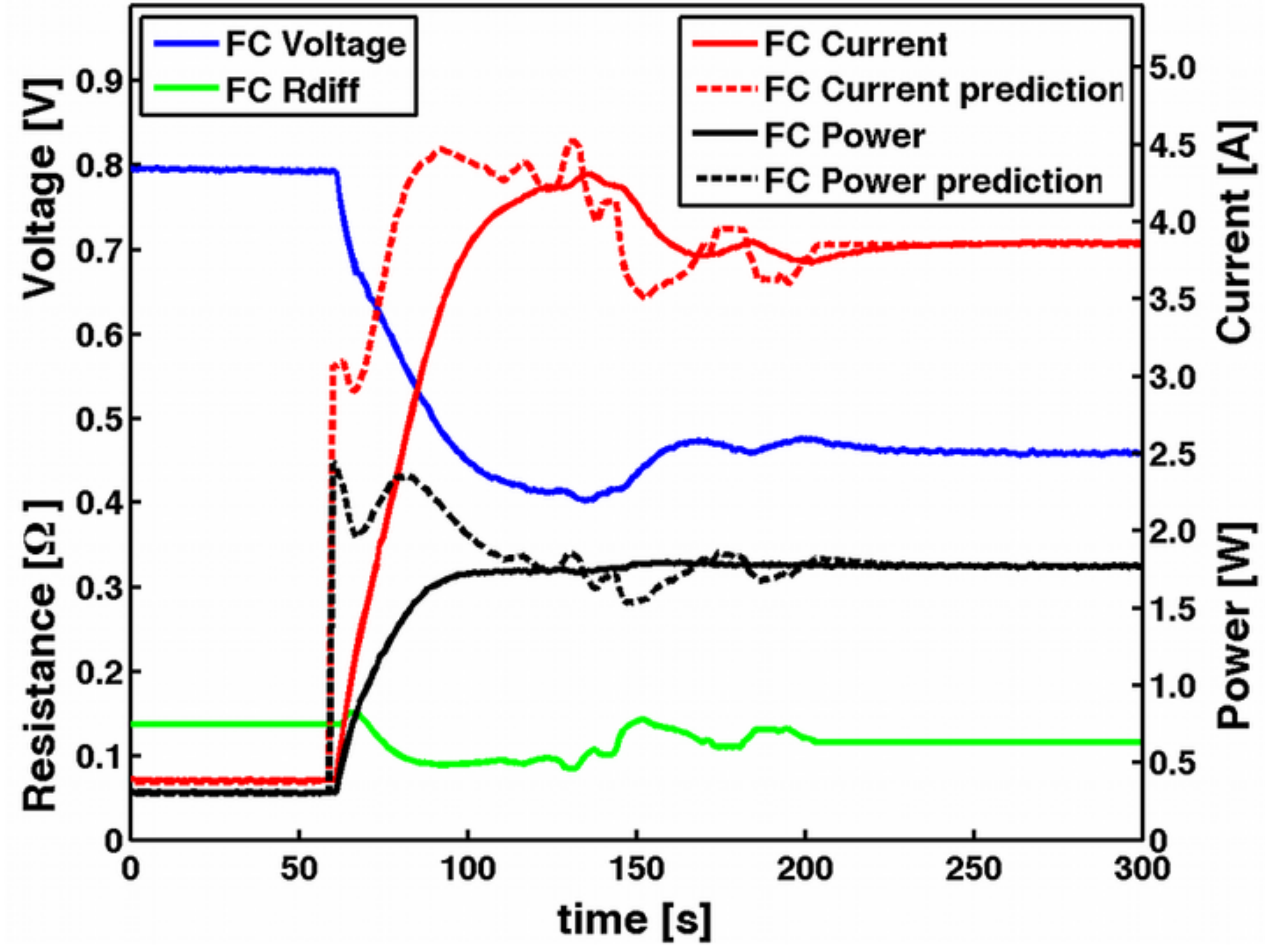




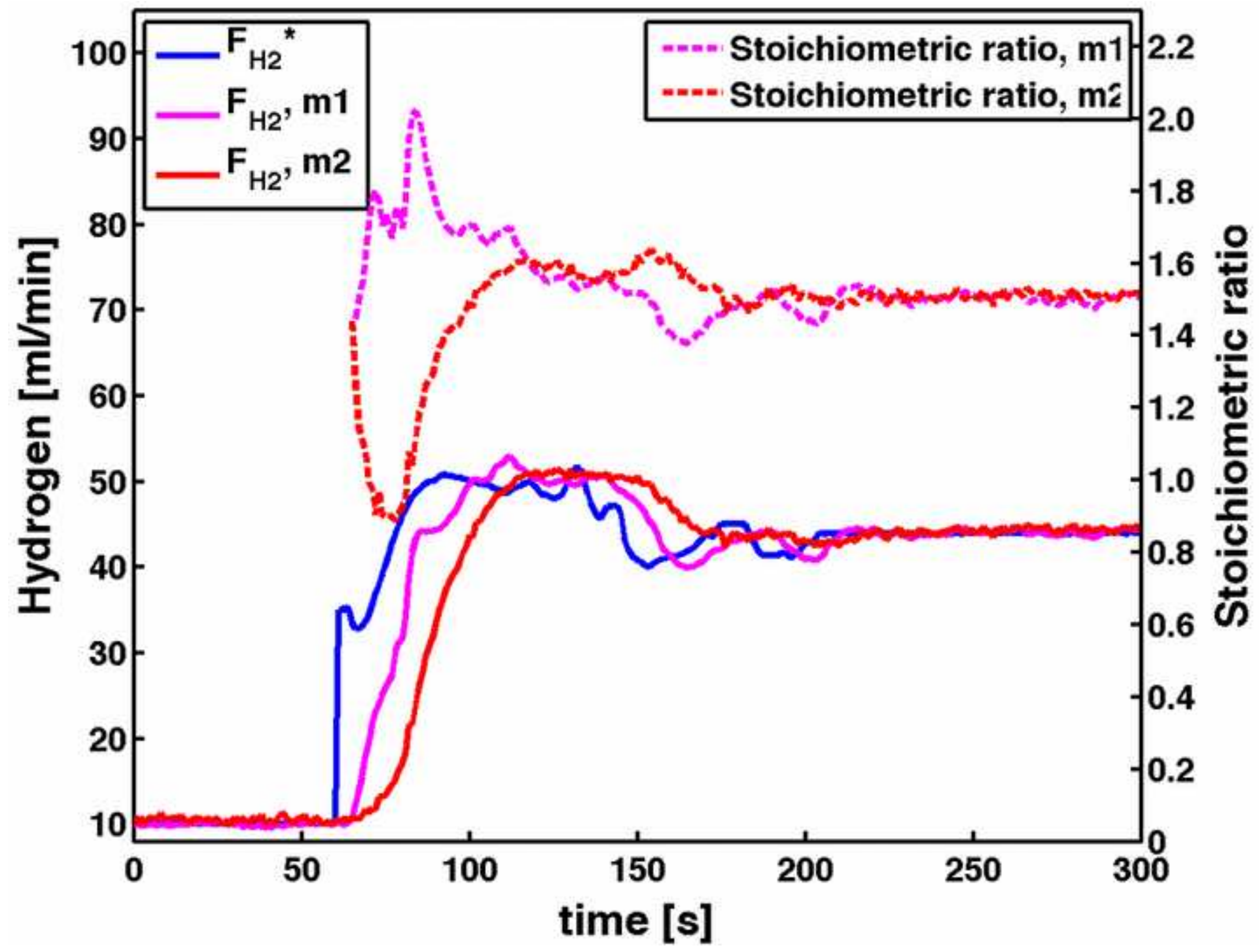




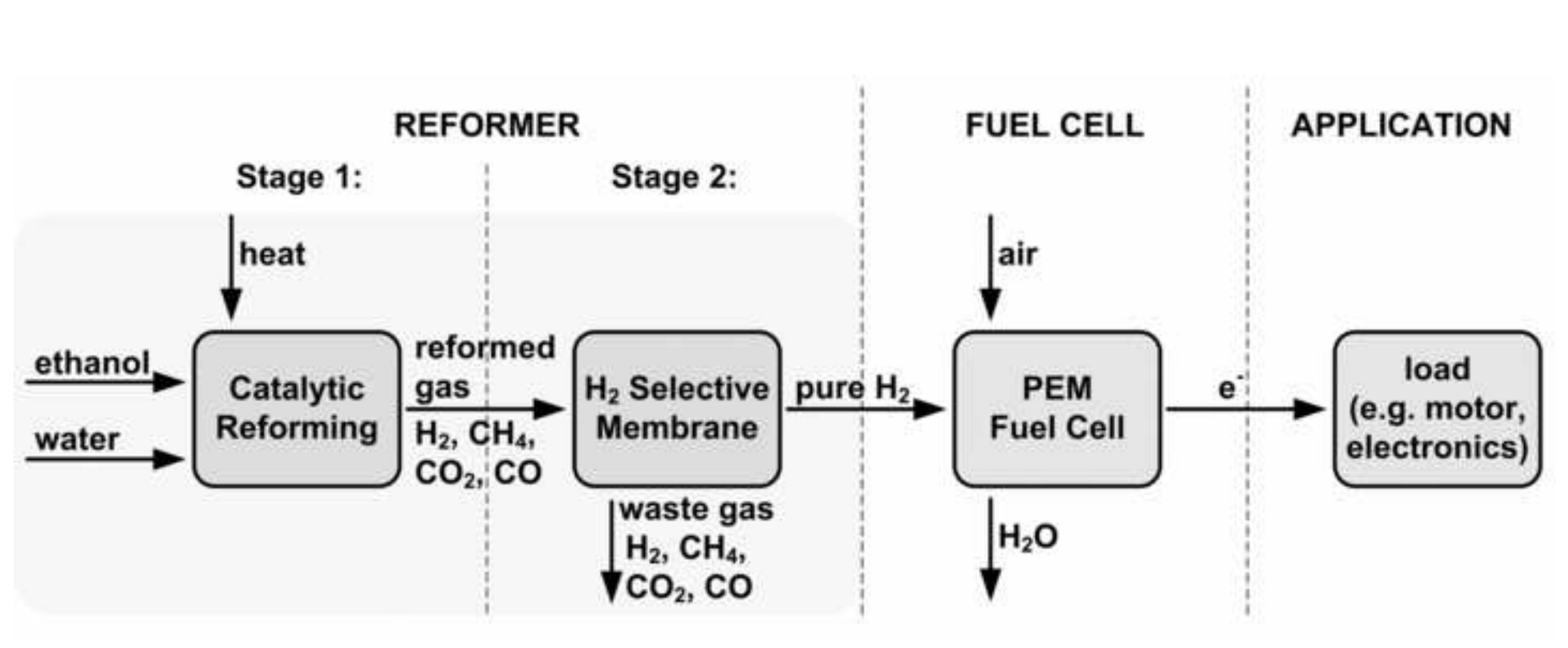

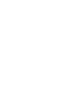



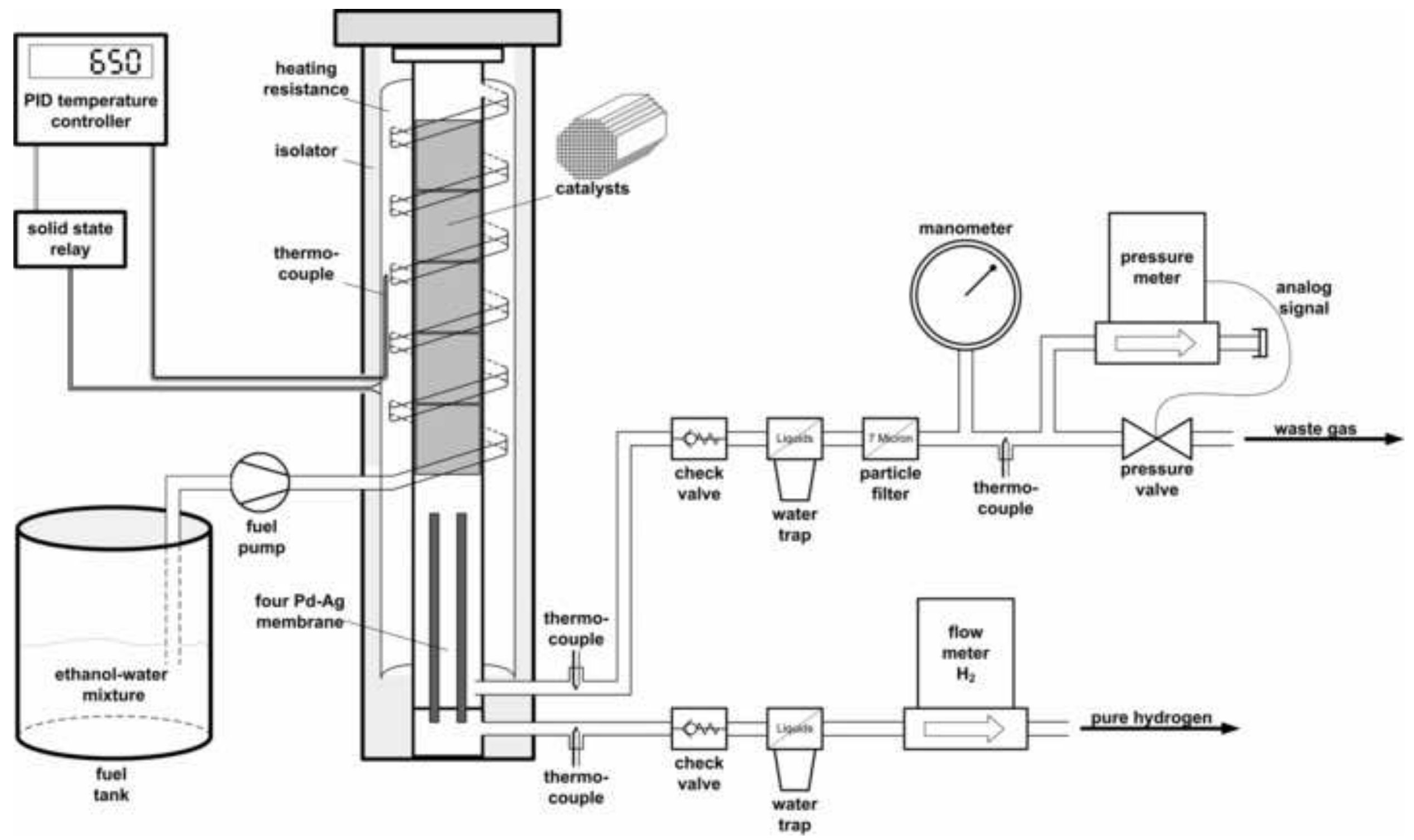


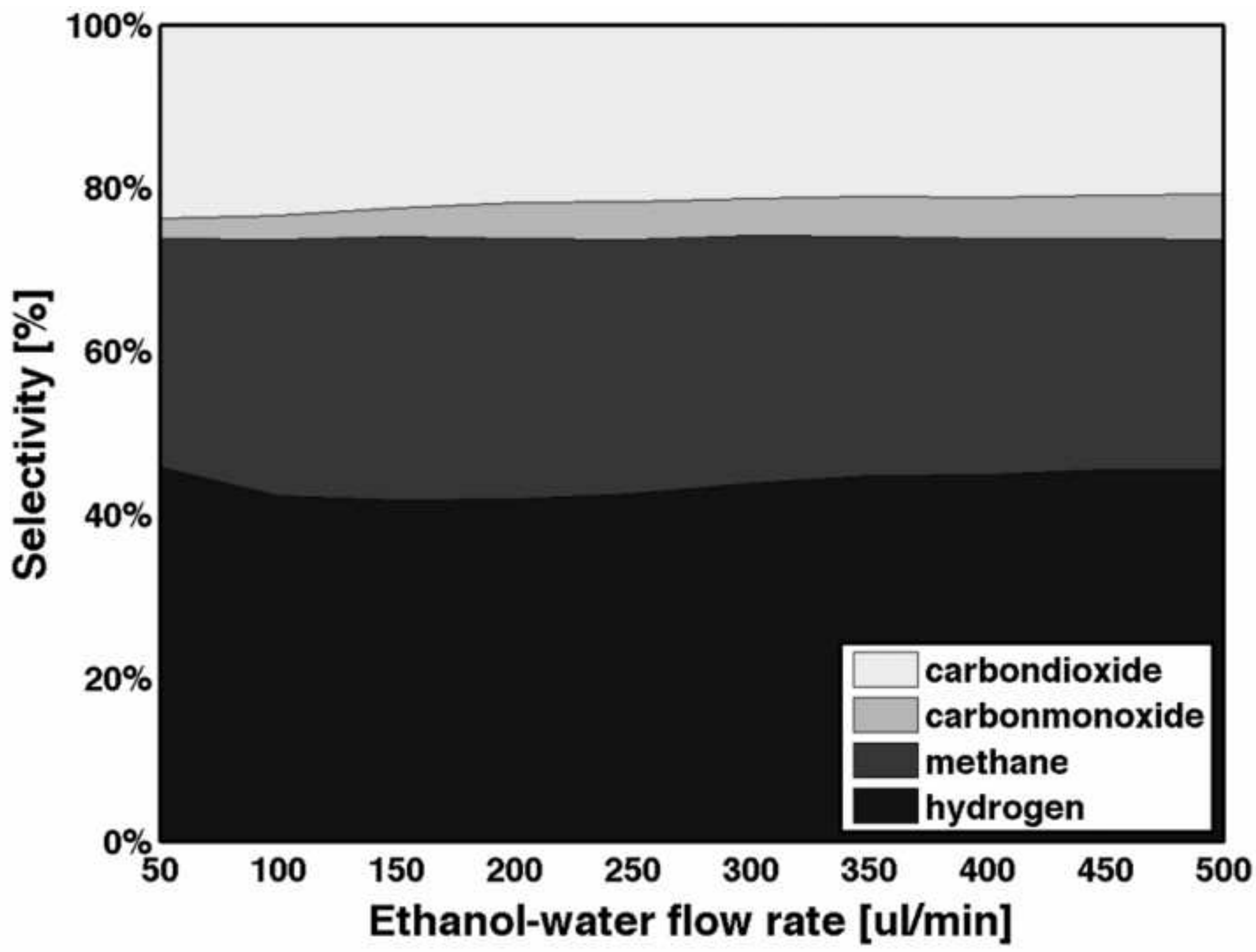




\section{$100 \%$}

$80 \%$

$60 \%$

$40 \%$

\$

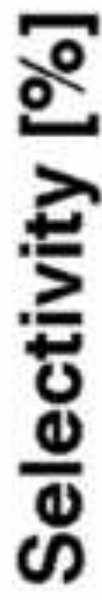

$20 \%$

$0 \%$

46

8

10

12

\section{Pressure [bar]}

carbondioxide carbonmonoxide methane hydrogen

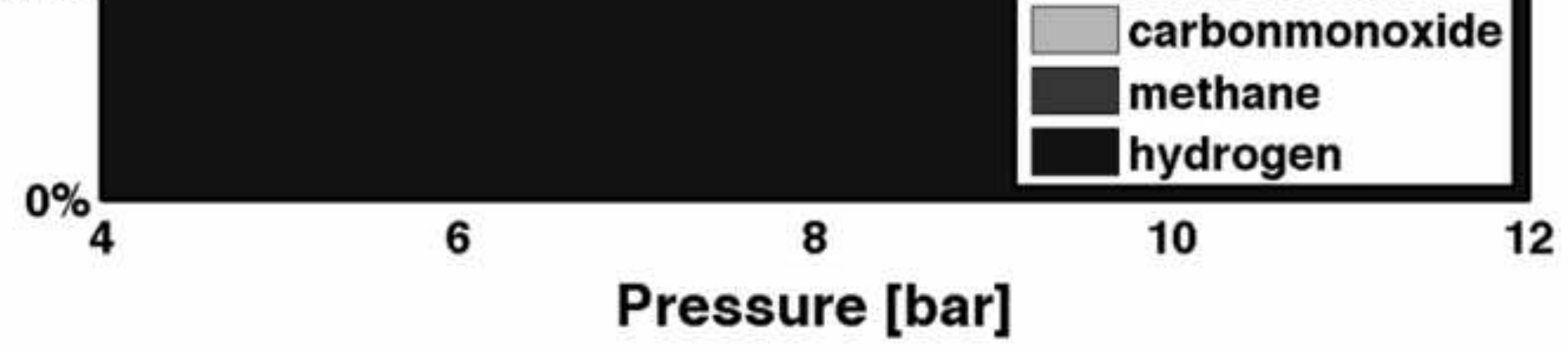




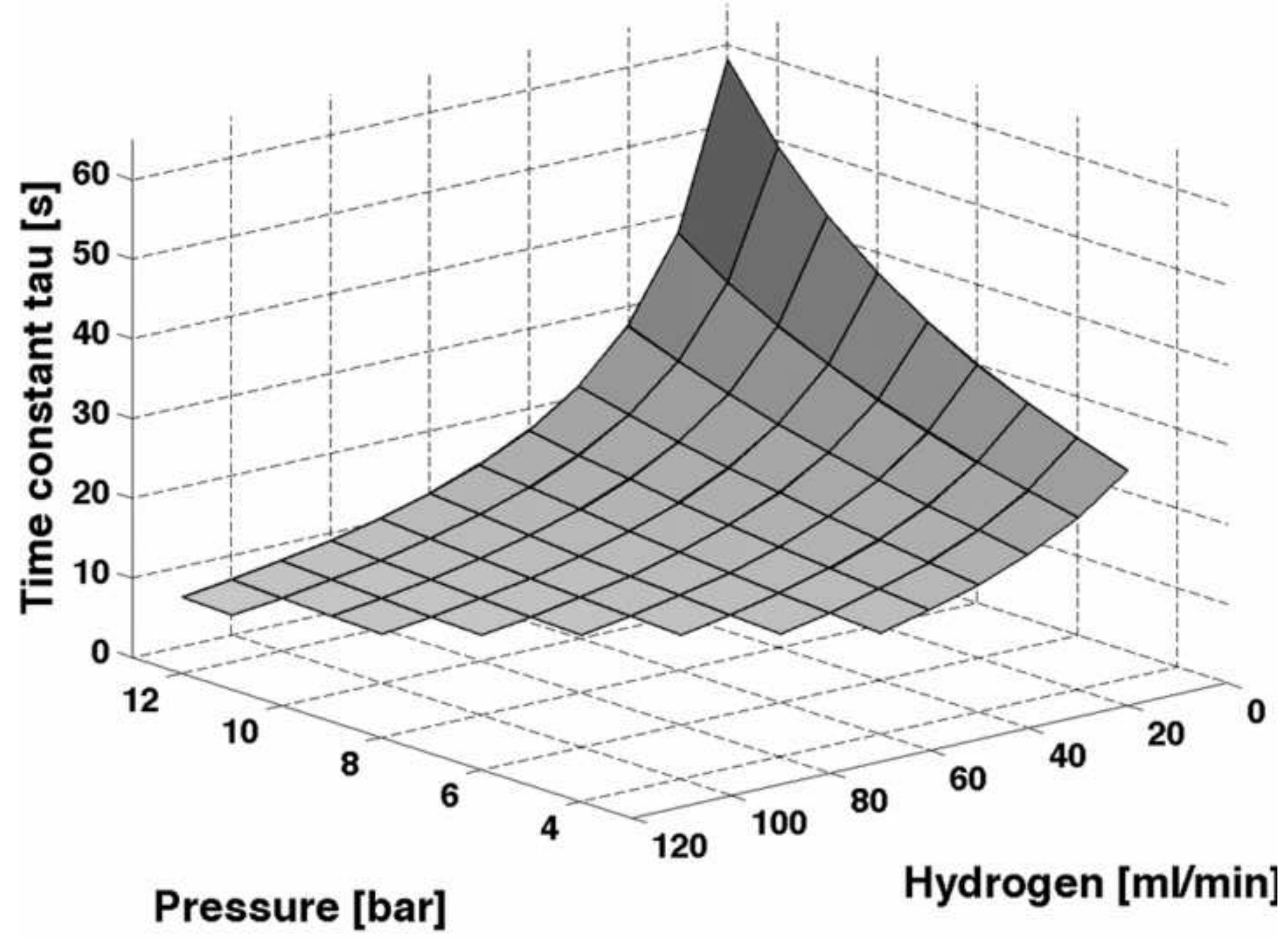




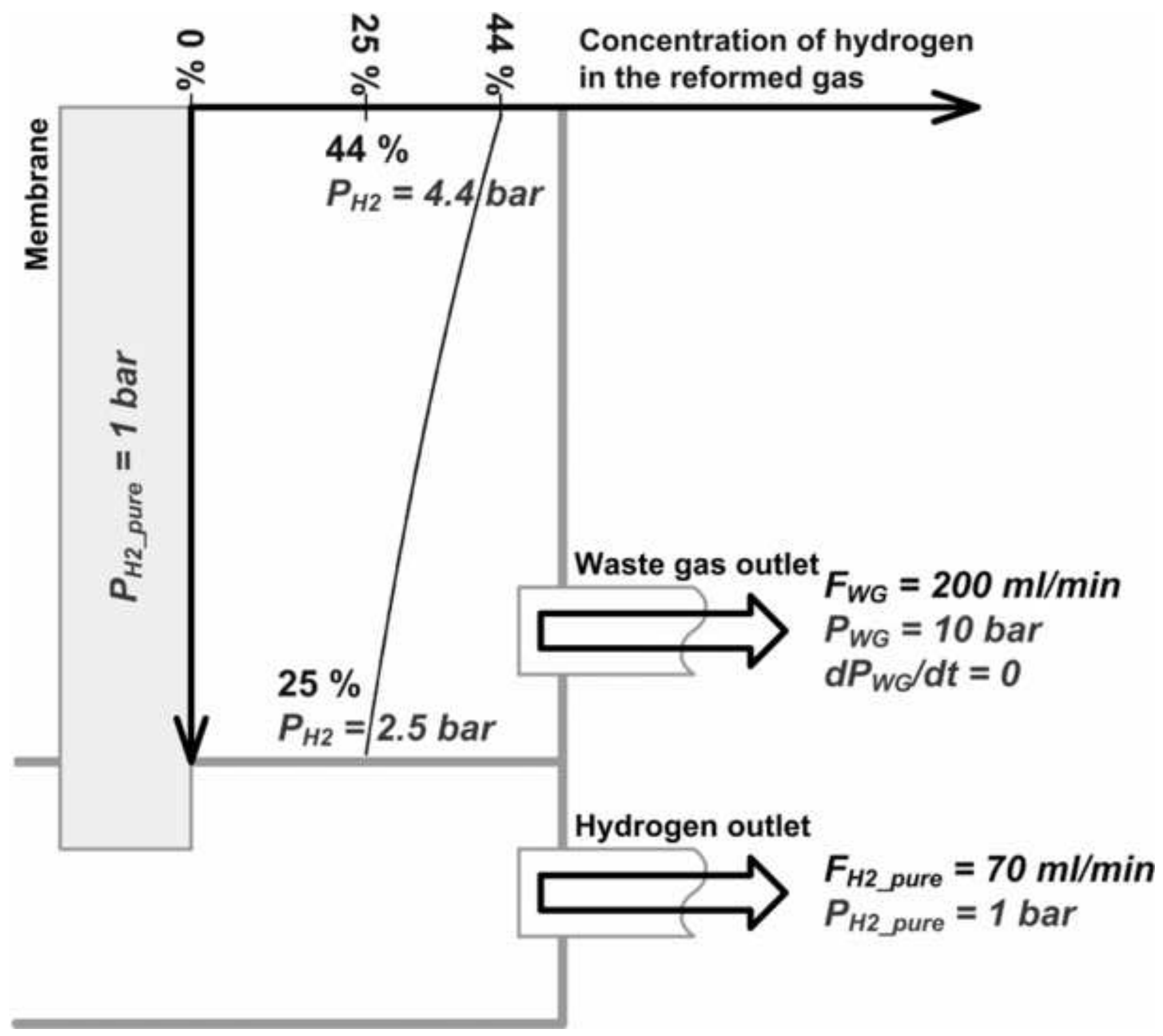




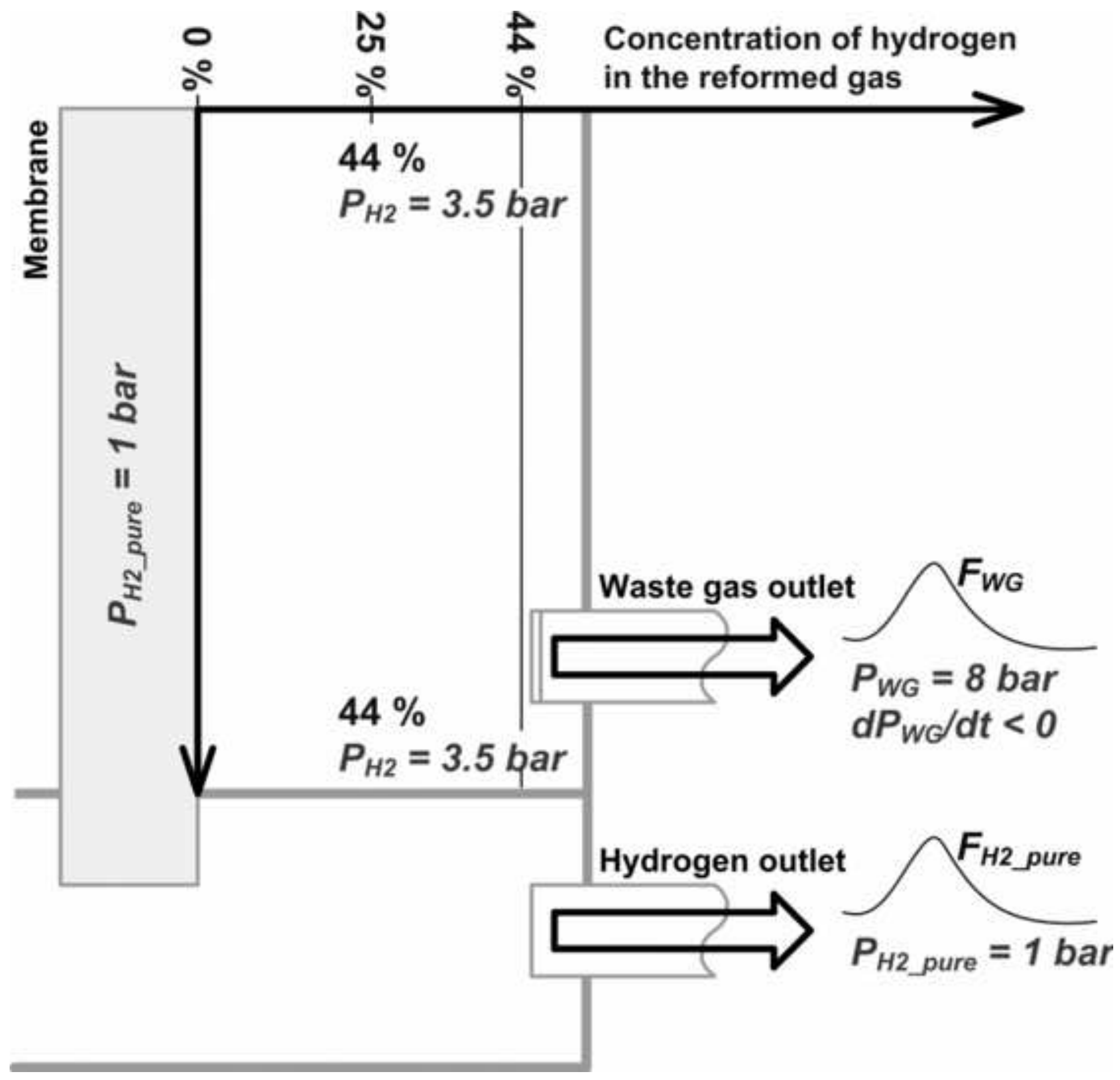


Fig7 Example for hydrogen peak b\&w

Click here to download high resolution image

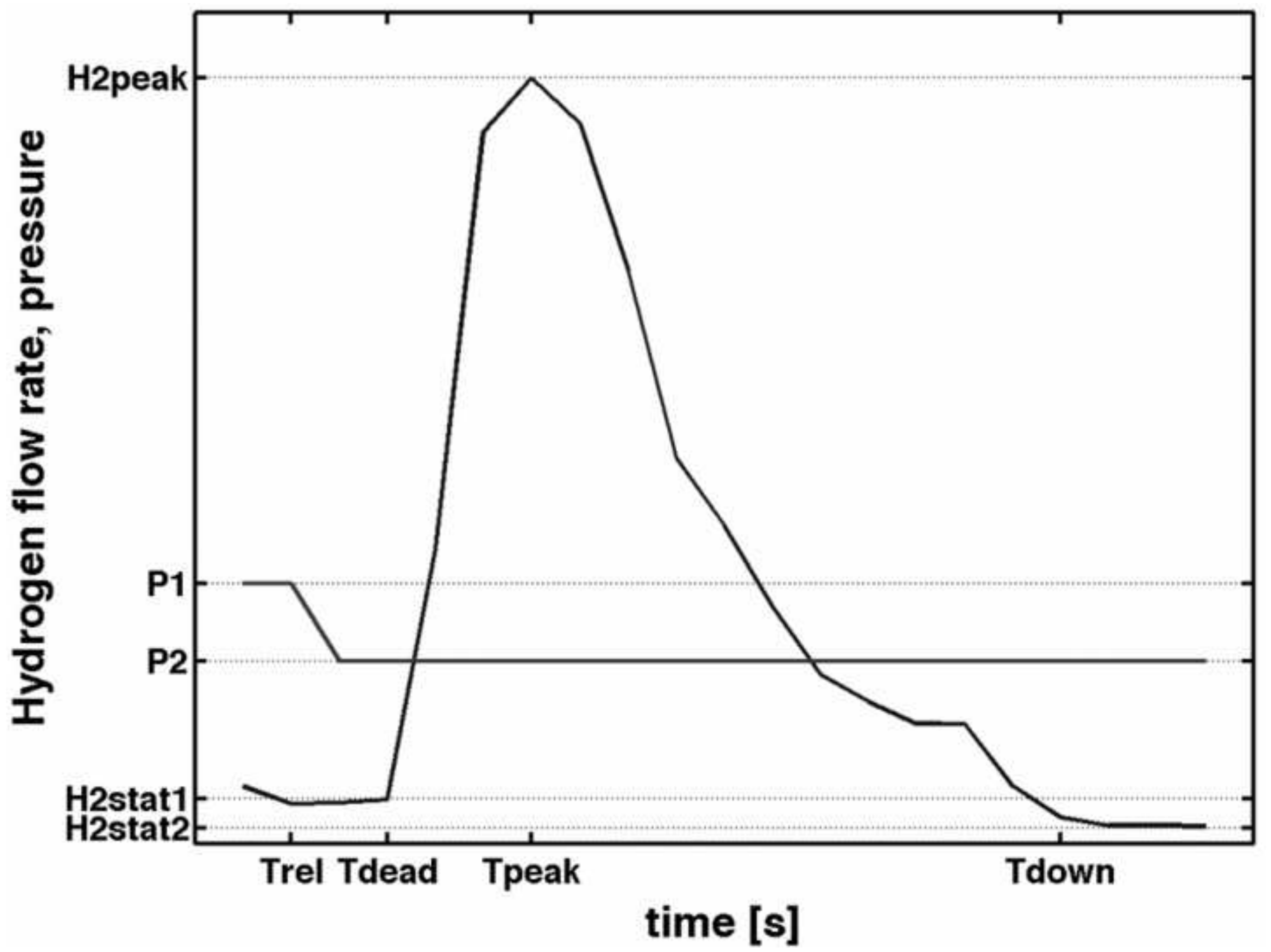




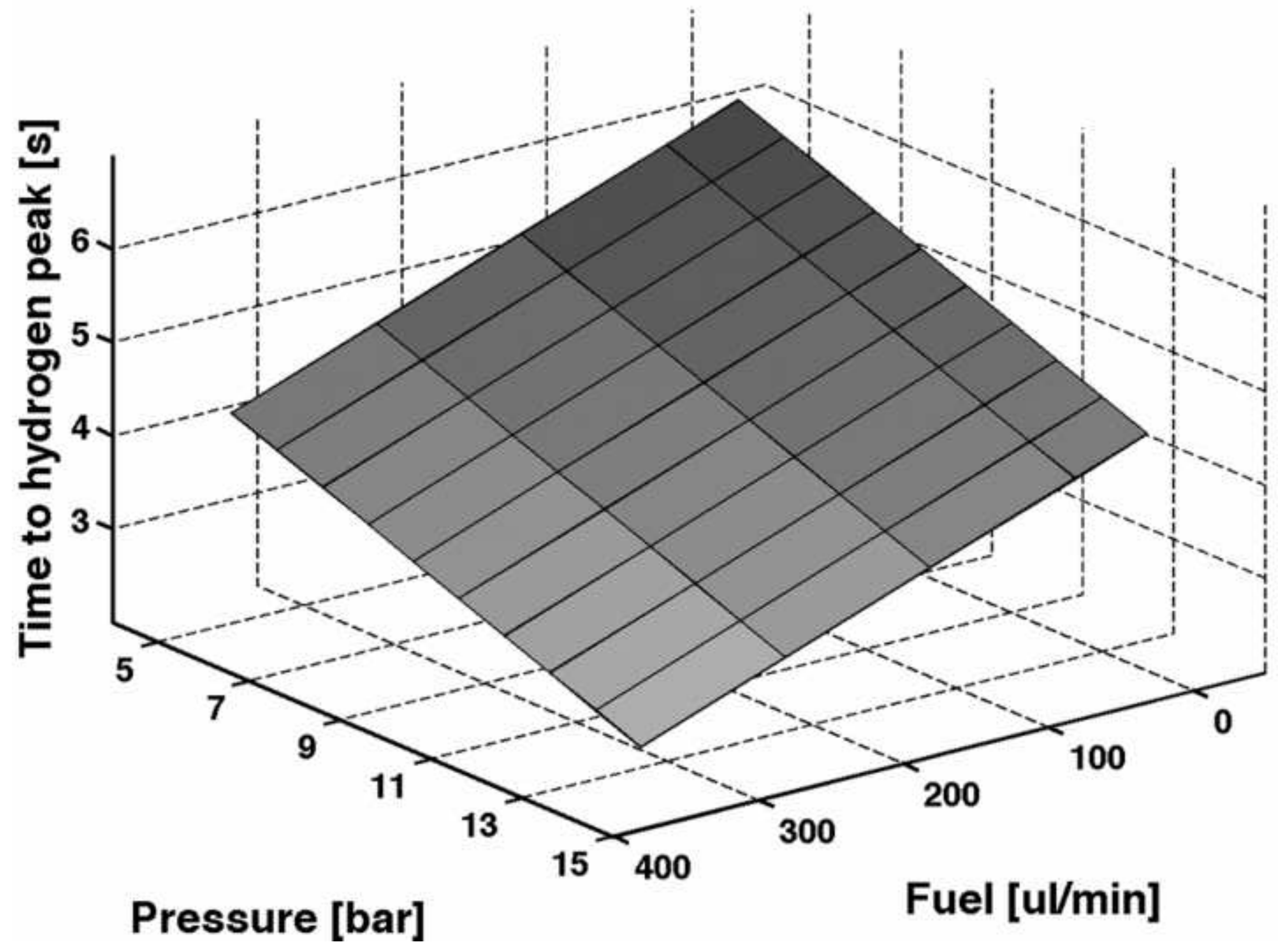




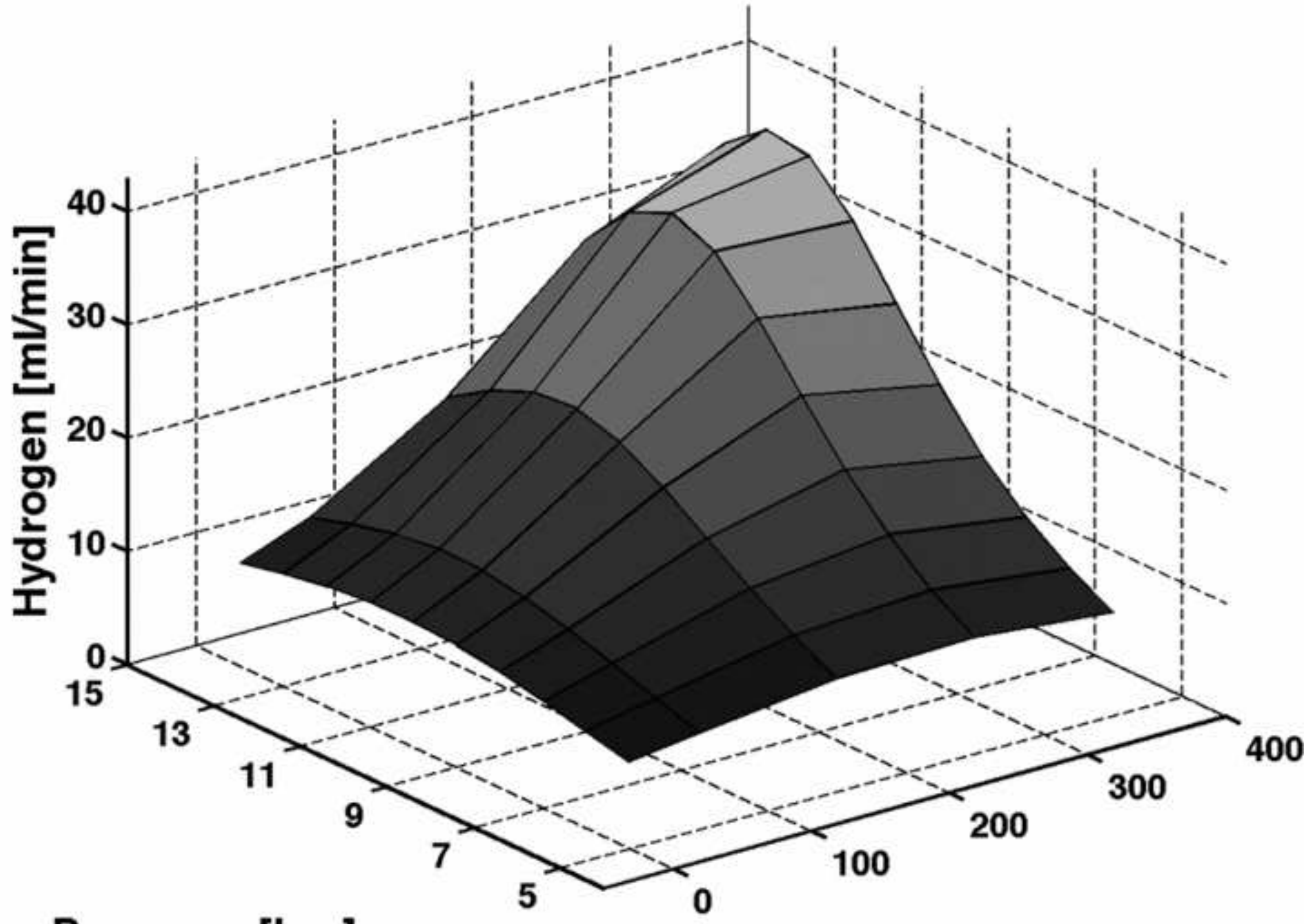

Pressure [bar]

Fuel [ul/min] 


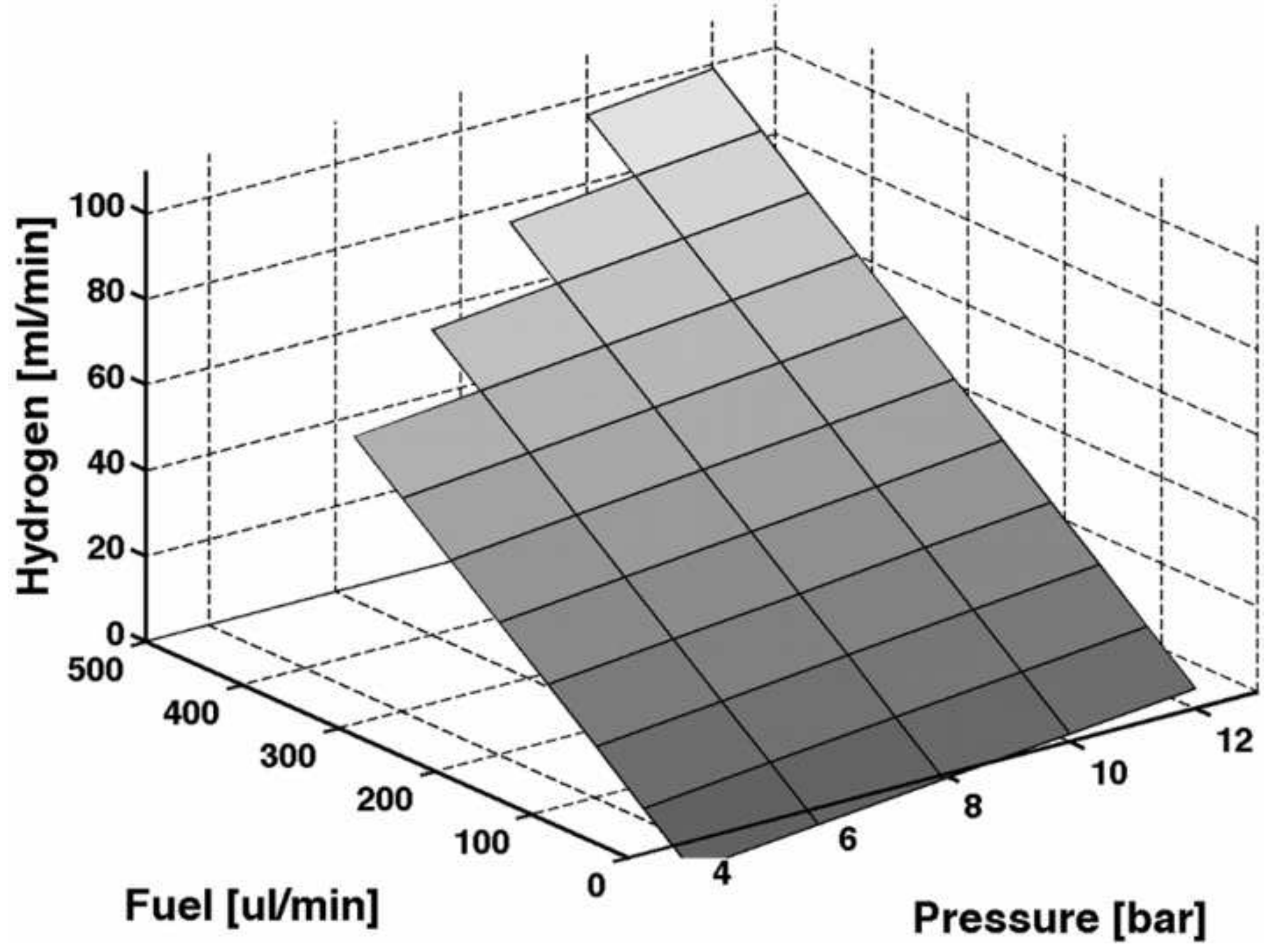




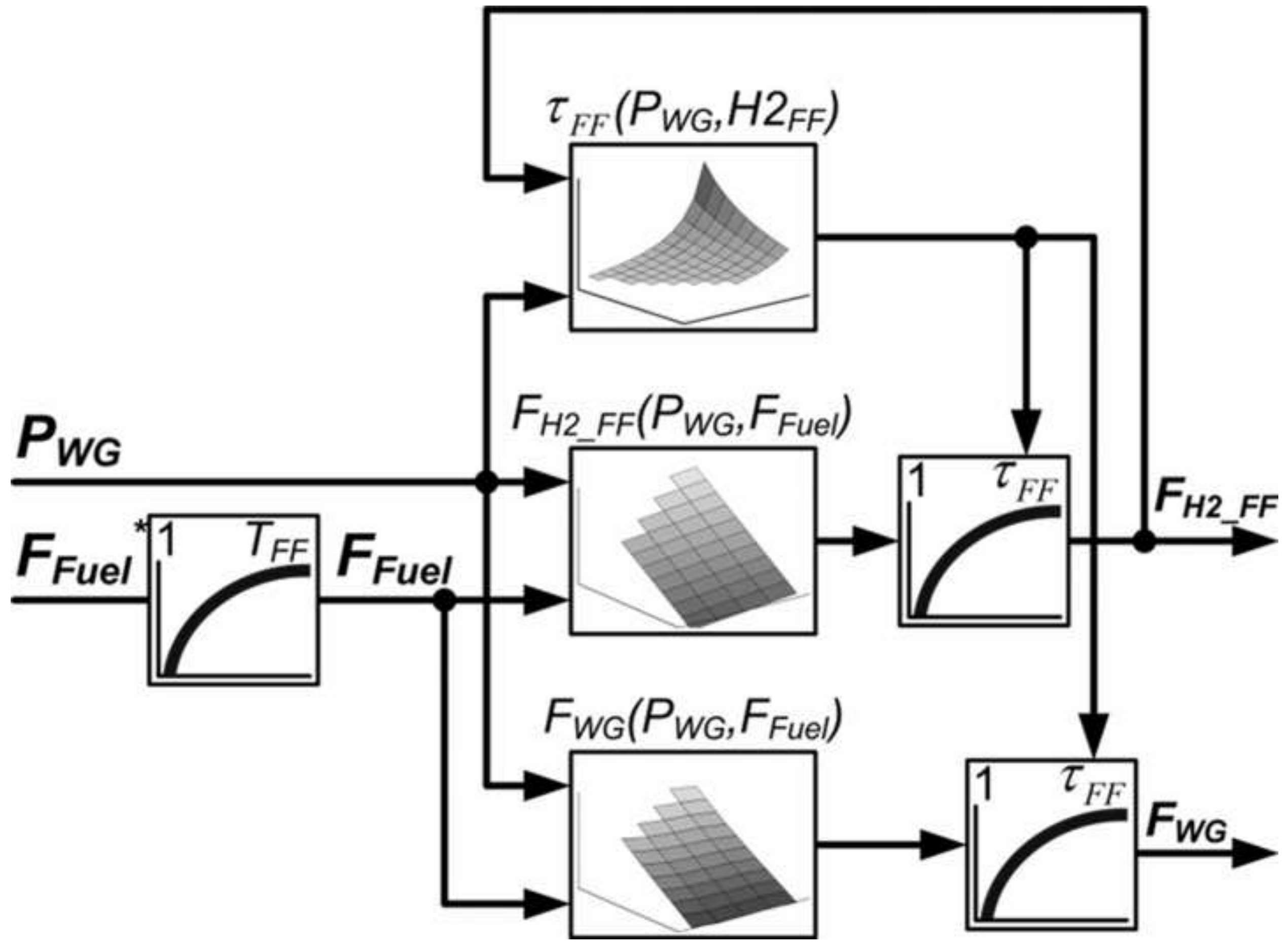


Fig12 Reformer sub-model: pressure response b\&w

Click here to download high resolution image

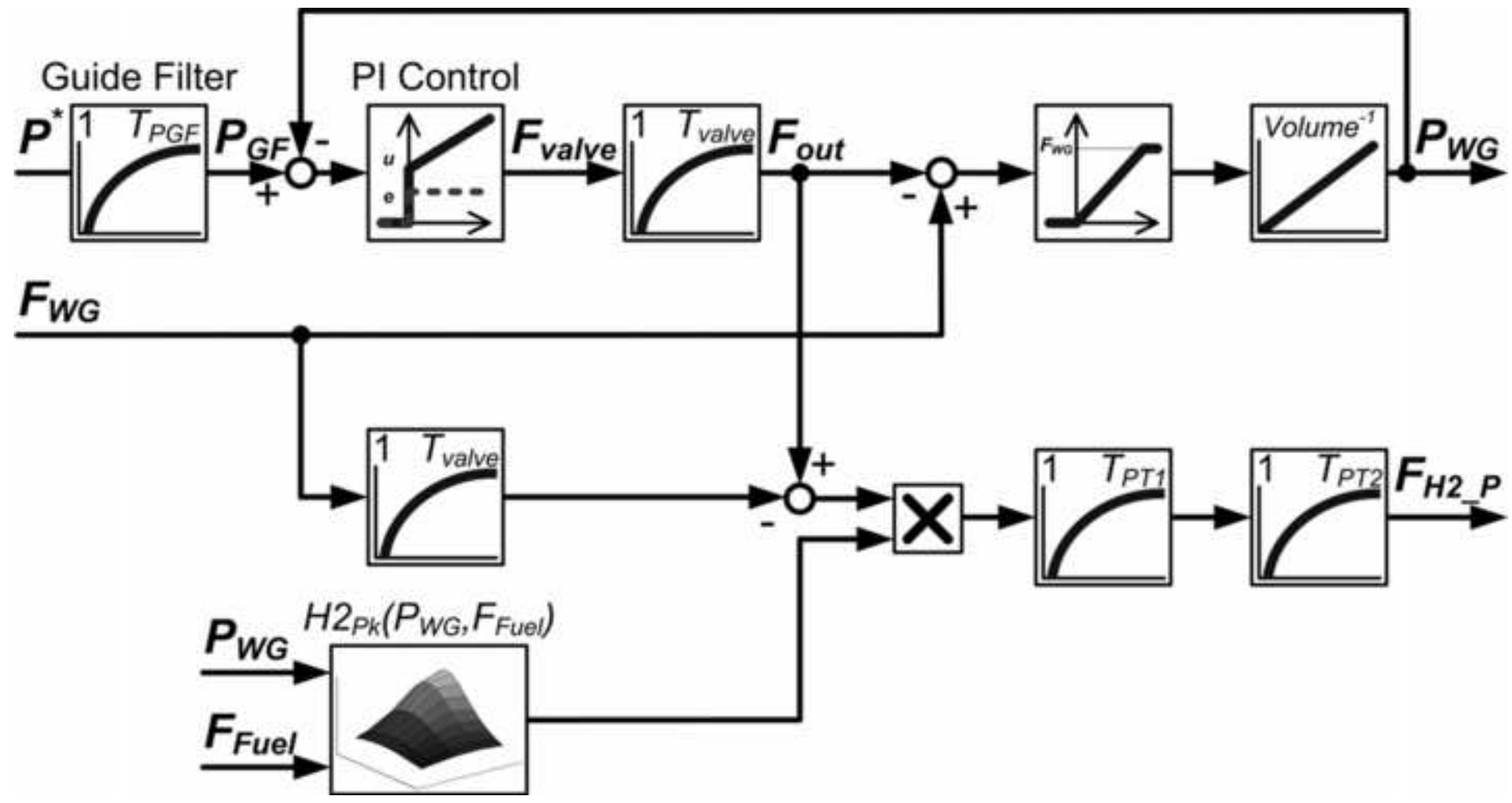


Fig13a Controller action: fuel flow rate b\&w

Click here to download high resolution image

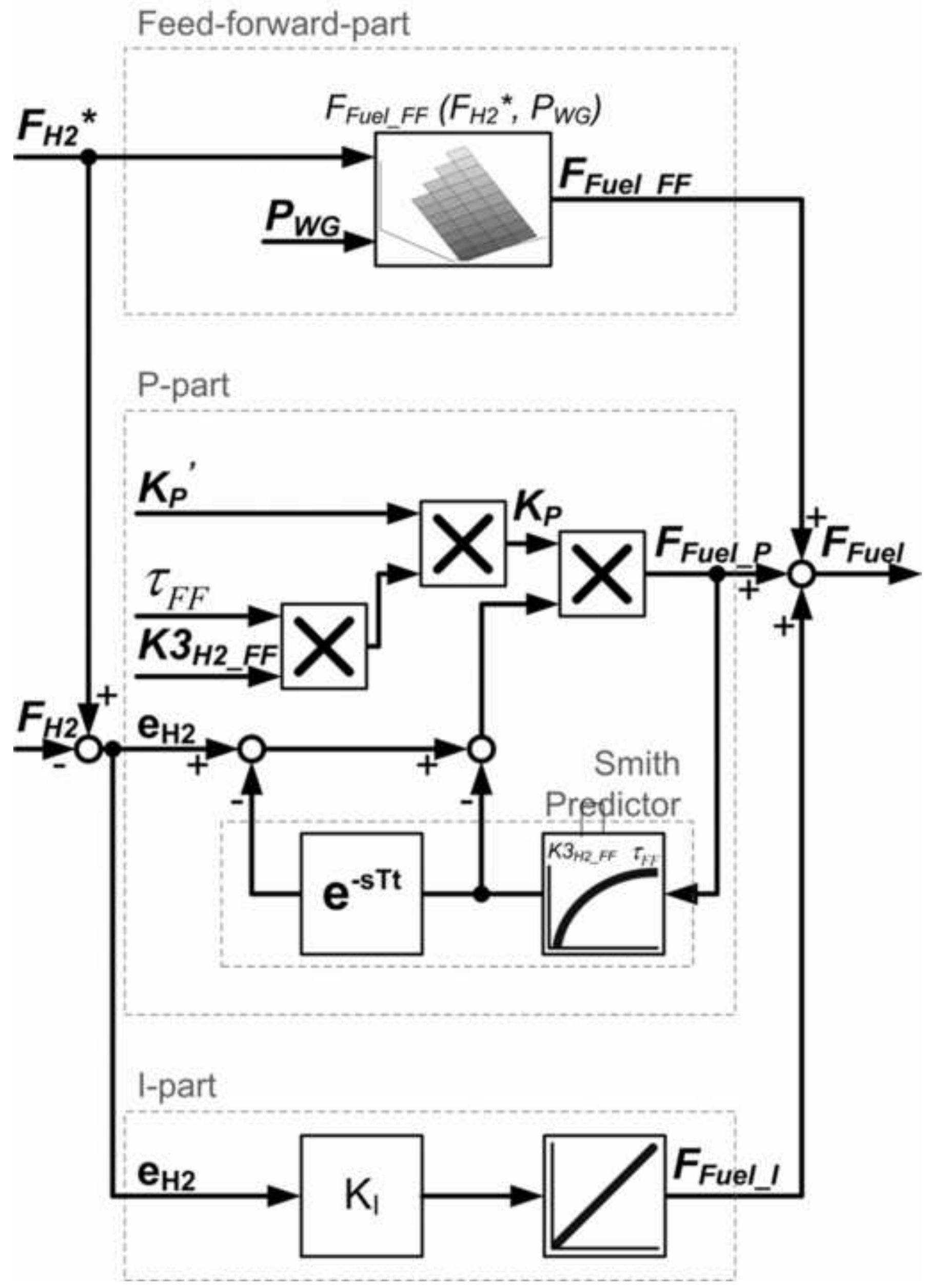


Predictive Control

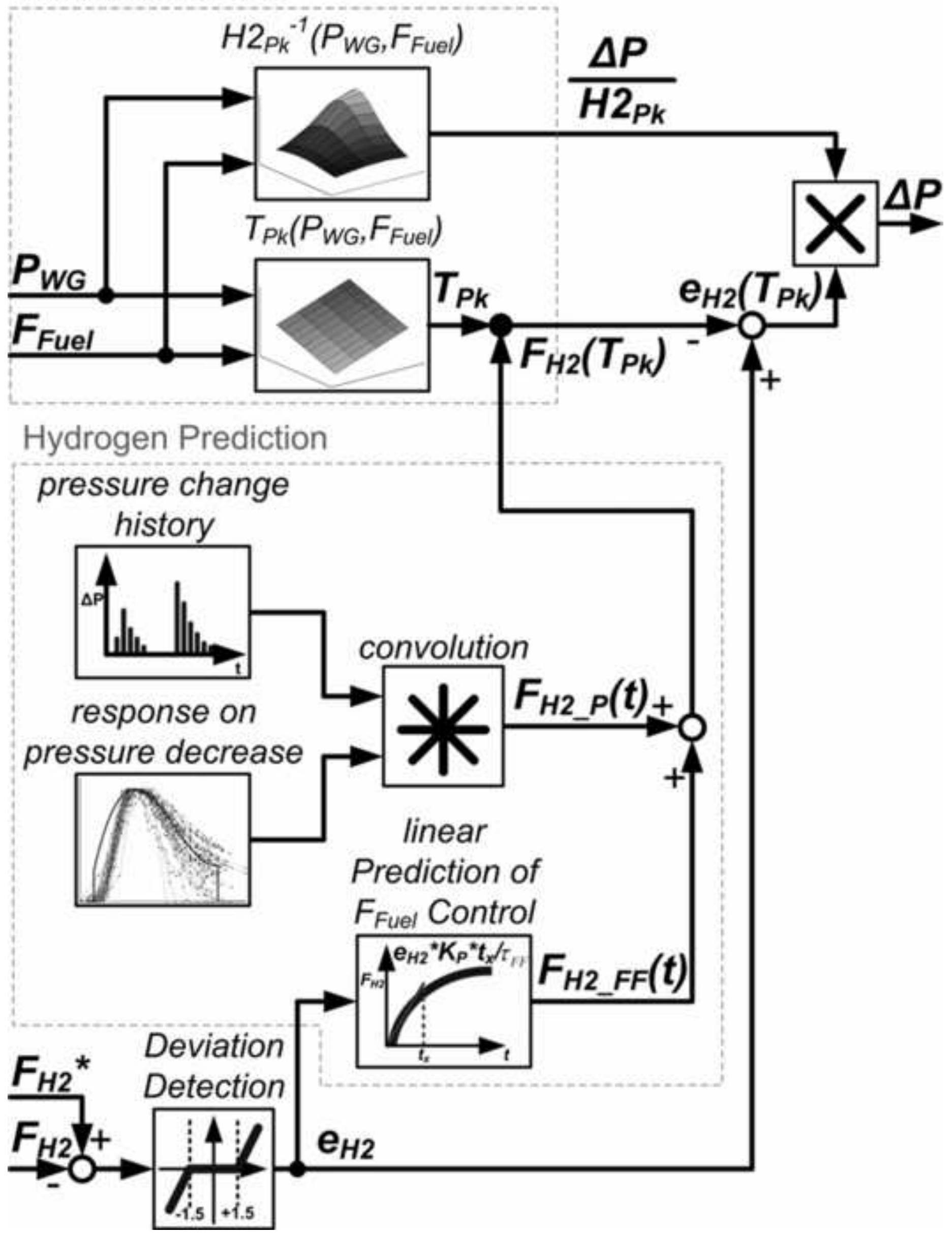



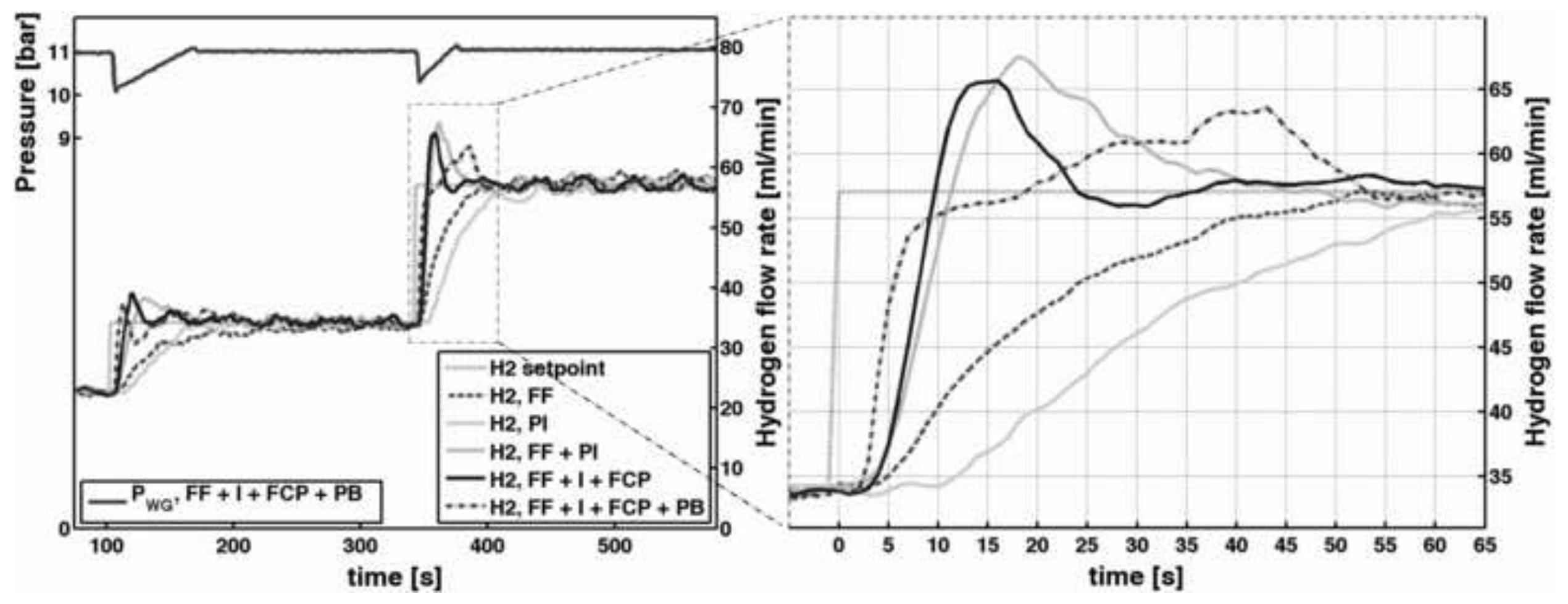


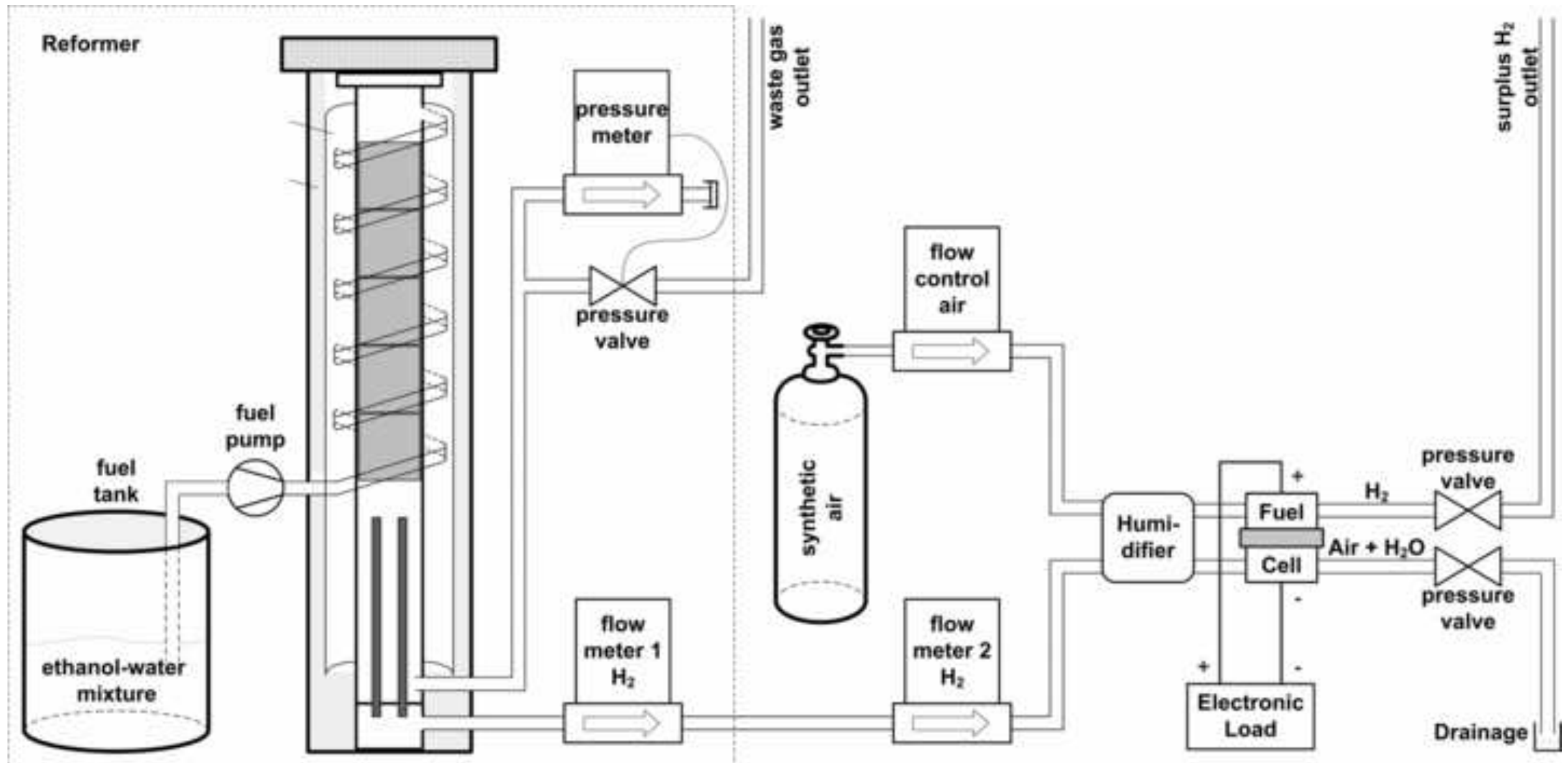




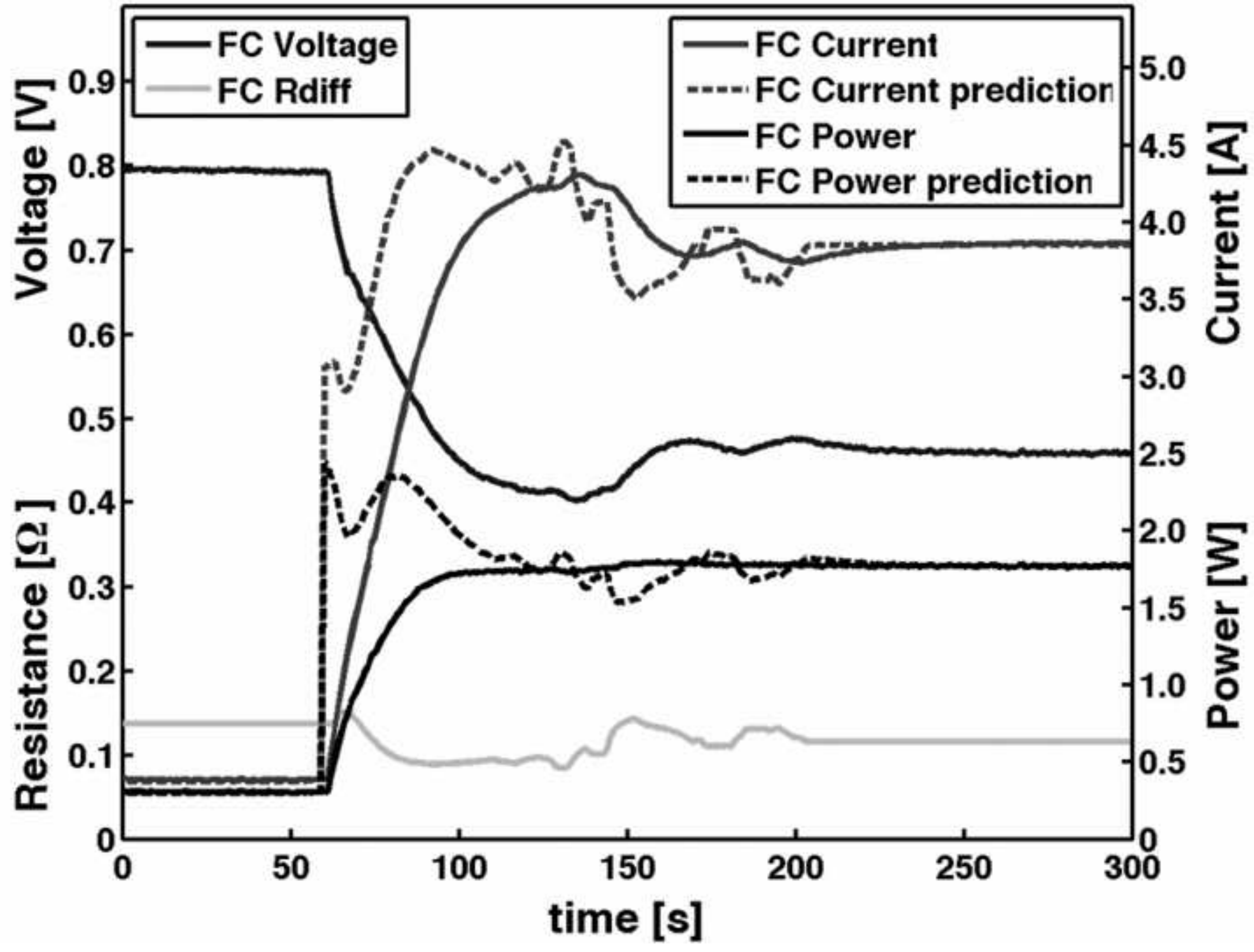




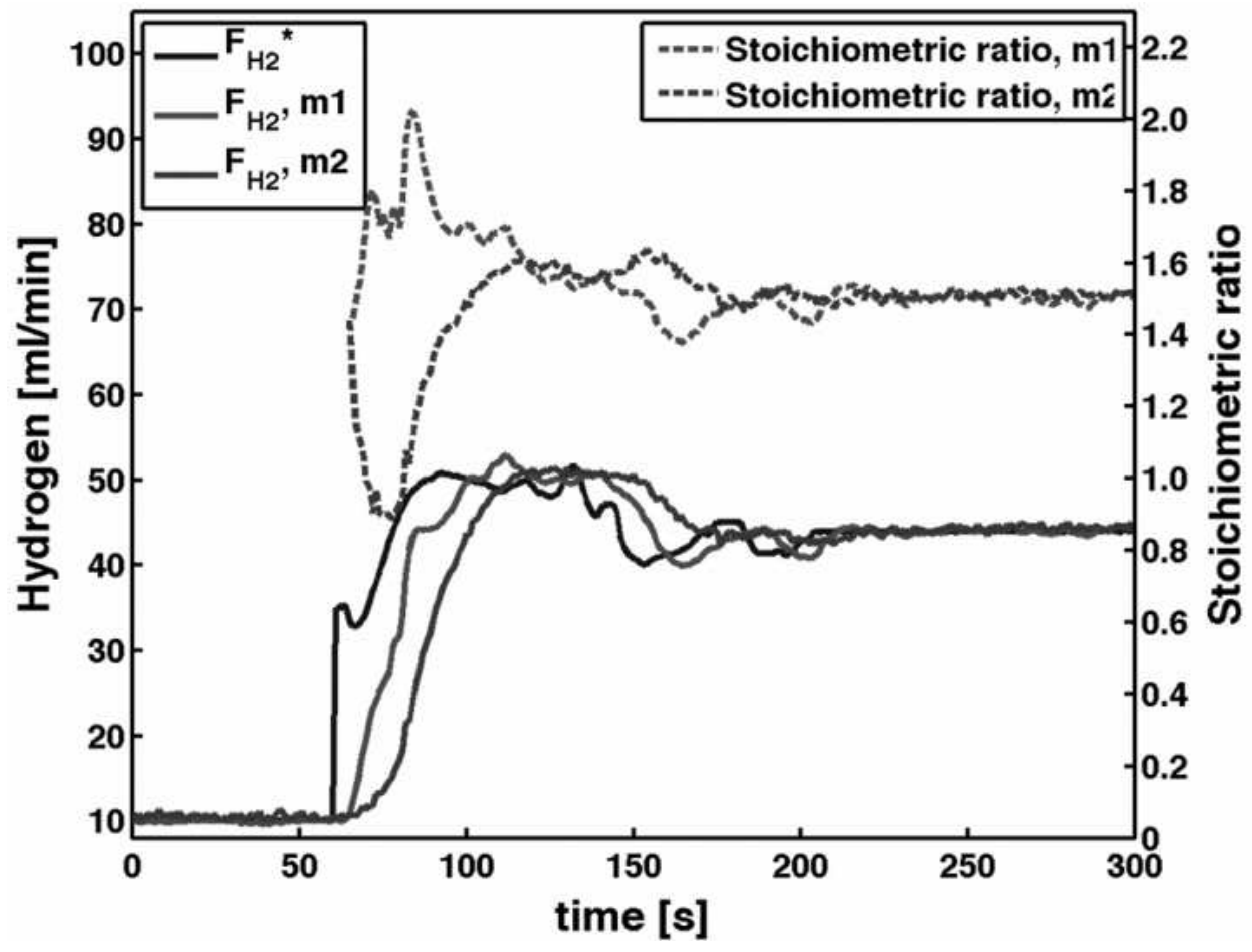

